Florida International University FIU Digital Commons

9-9-2014

\title{
Analysis of White Latex Paints using Laser Induced Breakdown Spectroscopy for Forensic Applications
}

Melissa Zwilling

Florida International University, mzwil001@fiu.edu

DOI: $10.25148 /$ etd.FI14110715

Follow this and additional works at: https://digitalcommons.fiu.edu/etd

Part of the Analytical Chemistry Commons

\section{Recommended Citation}

Zwilling, Melissa, "Analysis of White Latex Paints using Laser Induced Breakdown Spectroscopy for Forensic Applications" (2014). FIU Electronic Theses and Dissertations. 1590.

https://digitalcommons.fiu.edu/etd/1590 


\section{FLORIDA INTERNATIONAL UNIVERSITY}

Miami, FL

\section{ANALYSIS OF WHITE LATEX PAINTS USING LASER INDUCED BREAKDOWN SPECTROSCOPY FOR FORENSIC APPLICATIONS}

A thesis submitted in partial fulfillment of the

requirements for the degree of

MASTER OF SCIENCE

in

CHEMISTRY AND BIOCHEMISTRY

by

Melissa Zwilling

2014 
To: Interim Dean Michael Heithaus

College of Arts and Sciences

This thesis, written by Melissa Zwilling, and entitled Analysis of White Latex Paints Using Laser Induced Breakdown Spectroscopy for Forensic Applications, having been approved in respect to style and intellectual content, is referred to you for judgment.

We have read this thesis and recommend that it be approved.

Yong Cai

Bruce McCord

$\overline{\text { José Almirall, Major Professor }}$

Date of Defense: September 9, 2014

The thesis of Melissa Zwilling is approved.

Interim Dean Michael Heithaus

College of Arts and Sciences

Dean Lakshmi Reddi

University Graduate School

Florida International University, 2014 


\section{DEDICATION}

"And let your best be for your friend. If he must know the ebb of your tide, let him know its flood also. For what is your friend that you should seek him with hours to kill? Seek him always with hours to live."

-Kahlil Gibran

From, "The Prophet"

I would like to dedicate this work to the friends who have become my family.

Without their support, I would never have had the strength to finish this research. 


\section{ACKNOWLEDGMENTS}

First and foremost, I must thank my major professor, Dr. José Almirall. I have little doubt that if he had not accepted me into his research group, I would never have completed my degree. His guidance over the years has consistently led me towards the finish line, and for that I am extremely grateful.

I would also like to thank Dr. Yong Cai for not only serving on my committee, but for his advice about navigating through graduate school. His input in both personal advice and professional academic opinion has been invaluable.

Additional thanks to Dr. Bruce McCord for serving on my committee and helping to guide me through this process.

I must also acknowledge the Presidential Enhanced Assistantship, which it has been a great honor to receive.

Most importantly, I want to thank the friends who have become my family. There are many people who can count themselves on this list, and many who will be on it in the future, but I will focus on the few individuals who have most impacted my life.

In college, I was lucky enough to meet Eugene Malinskiy, who has stuck with me through the worst times of my life. There are very few people who would have had the patience and courage to support me through those times, and it is difficult to describe how fortunate I feel that I can count Eugene as one of my best friends. He introduced me to what true friendship really is, opened my eyes to the wider world around me, and has continued to show his support even over a thousand miles away in Ohio. I know that I can rely on him for advice not only on research, but on life in general. Thank you. 
Finally, I want to thank Alexander Landera. His love has helped carry me through all my hard years of graduate school. He encouraged me when I thought there was no hope, he supported me when I thought I would have to fight alone, and he patiently comforted me regardless of how many times anxiety took hold. I've also had the joy of venturing out to new experiences with him, discussing the finer points of topics ranging from philosophy to football, and had the honor of being accepted by his family. His patience with me was certainly one of the keys to my own success. This research would never have been completed without him, and I would never have earned a graduate degree. One paragraph in an acknowledgements section doesn't do justice to how much his strength, patience, and love has motivated me, but for now it will have to do. Alex, thank you for your unwavering support and love. 
ABSTRACT OF THE THESIS

\title{
ANALYSIS OF WHITE LATEX PAINTS USING LASER INDUCED BREAKDOWN SPECTROSCOPY FOR FORENSIC APPLICATIONS
}

\author{
by \\ Melissa Zwilling \\ Florida International University, 2014 \\ Miami, Florida \\ Professor José Almirall, Major Professor
}

The analysis of white latex paint is a problem for forensic laboratories because of difficulty in differentiation between samples. Current methods provide limited information that is not suitable for discrimination. Elemental analysis of white latex paints has resulted in 99\% discriminating power when using LA-ICP-MS; however, mass spectrometers can be prohibitively expensive and require a skilled operator. A quick, inexpensive, effective method is needed for the differentiation of white latex paints.

In this study, LIBS is used to analyze 24 white latex paint samples. LIBS is fast, easy to operate, and has a low cost. Results show that $98.1 \%$ of variation can be accounted for via principle component analysis, while Tukey pairwise comparisons differentiated $95.6 \%$ with potassium as the elemental ratio, showing that the discrimination capabilities of LIBS are comparable to those of LA-ICP-MS. Due to the many advantages of LIBS, this instrument should be considered a necessity for forensic laboratories. 


\section{TABLE OF CONTENTS}

CHAPTER

PAGE

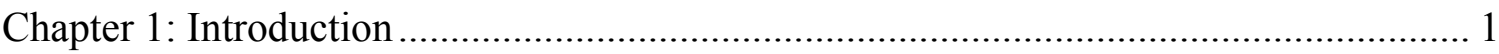

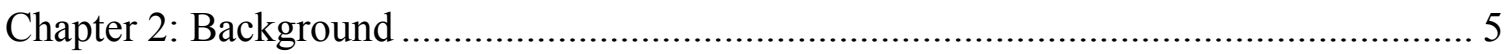

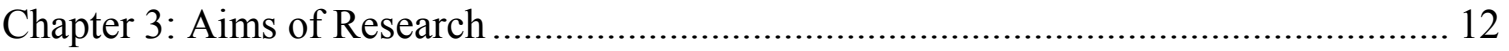

Chapter 4: Experimental Method Development ........................................................... 14

a. Chapter 4-A: Optimization of LIBS Parameters with White Latex Paint......... 14

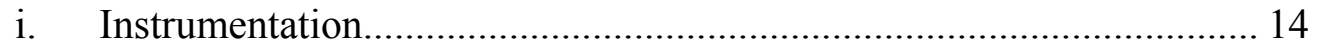

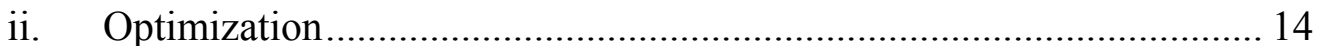

b. Chapter 4-B: Previously Analyzed Paint Samples ........................................ 20

c. Chapter 4-C: Determination of LIBS Discrimination Capabilities .................. 26

Chapter 5: Results and Discussion........................................................................... 33

d. Chapter 5-A: PCA of Gagnon Paint Samples Analyzed via LIBS ................... 33

e. Chapter 5-B: Tukey's Pairwise Analysis of Gagnon Paint Samples Analyzed by LIBS ................................................................................ 41

f. Chapter 5-C: Comparison of Discrimination Power Between LIBS and LA-

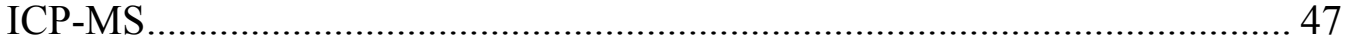

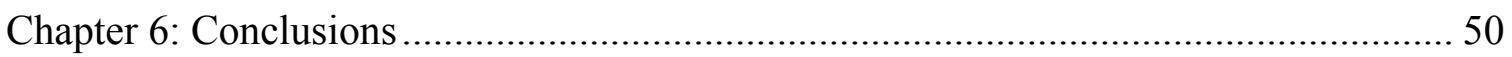

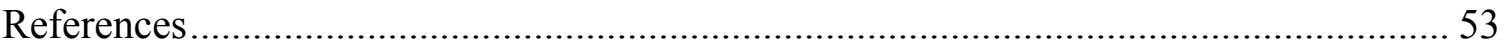




\section{LIST OF TABLES}

TABLE

PAGE

Table 4.1 Optimization parameters for LIBS on Behr 8050 white latex paint ............... 15

Table 4.2 Paint analysis parameters ...................................................................... 23

Table 4.3 A list of examined paint samples from Gagnon ......................................... 24

Table 4.4 The element menu for LIBS analysis .................................................. 25

Table 5.1 The percent account for variation using $\mathrm{K}$, Al, and $\mathrm{K}$ elemental ratios ........... 33

Table 5.2 Tukey pairwise comparison between two GLD 2013 samples....................... 36

Table 5.2 The pairwise Tukey's test for K elemental ratio......................................... 45

Table 5.3 The pairwise Tukey's test for Al elemental ratio .......................................... 46

Table 5.4 Pairs that could not be distinguished via Tukey's pairwise analysis ............... 47

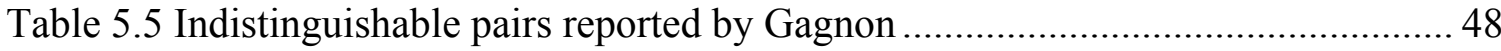




\section{LIST OF FIGURES}

FIGURE

PAGE

Figure 2.1 A diagram of a LIBS instrumental setup ............................................. 6

Figure 2.2 The intensity of optical signal decreases over time .................................... 7

Figure 4.1 Variation in signal-to-noise ratio with change in laser power....................... 17

Figure 4.2 Variation in signal-to-noise ratio with change in gate delay ........................ 18

Figure 4.3 Variation in signal-to-noise ratio with change in frequency ........................ 18

Figure 4.4 Variation in signal-to-noise ratio with change in gas flow rate..................... 19

Figure 4.5 Variation in signal-to-noise ratio with change in number of shots per spot.... 20

Figure 4.6 A typical crater from LIBS paint analysis ............................................. 26

Figure 4.7 Raw intensities of elemental ratio candidates ........................................ 28

Figure 4.8 Trends in elemental signal between LIBS and LA-ICP-MS data .................. 32

Figure 5.1 A 3-D PCA plot of all paint samples utilizing aluminum as the elemental

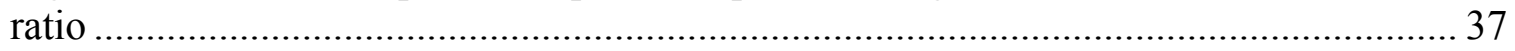

Figure 5.2 A 3-D PCA plot of paint samples including two replicates of GLD2013,

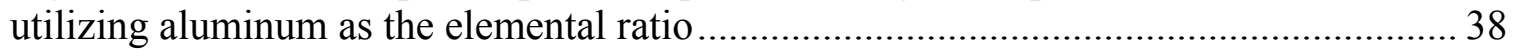

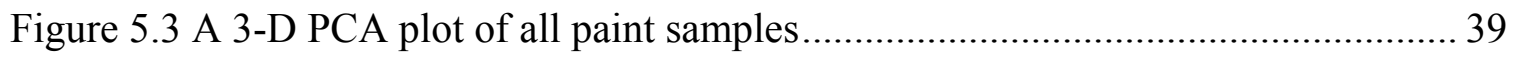

Figure 5.4 A 3-D PCA plot of paint samples including two replicates of GLD2013,

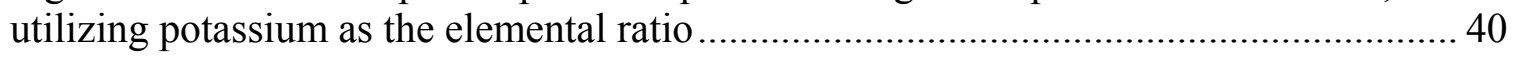

Figure 5.5 A visualization of the Tukey's pairwise comparison test for barium with potassium as an elemental ratio 


\section{Chapter 1: Introduction}

Forensic analyses are vitally important in modern criminal investigations. Crime laboratories receive samples ranging from glass, fibers, and blood splatter patterns to footprints, tire treads, and more. Chemical analyses of forensic samples are sometimes challenging due to the small sample sizes that are usually presented, such as a small paint chip or piece of glass from a car windshield. In these cases, visual identification is inadequate for verifying the origin of the sample, and chemical analyses, such as elemental analysis of trace metals, are employed.

Elemental analyses of forensic samples has already been successfully employed on such evidence as windshield glass [1], automotive paint [2, 3], paper [4], float glass, and gel ink. [5] Paints and coatings are common samples in forensic cases, which makes the ability to identify and differentiate paint origins an important tool. For example, architectural paint, or latex paint, can be presented in cases of forced entry (such as paint smears on a tool.) However, base coats for this type of paint are generally white and cannot be distinguished by color alone. This is a particular problem due to the commonality of white latex paint.

Paints and coatings have multiple components, but in the simplest form, latex paints possess a binder, resin, and pigment(s). The binder, also referred to as the vehicle, is used to ensure even distribution during the painting process and is typically an organic molecule. [3] The resin is a volatile component which allows the paint to dry, thereby changing the liquid form into a dry surface coating. Pigments, as the name suggests, are added to alter the colors of paints. Unlike binders and resins, pigments are often inorganic compounds that contain metallic elements. For example, titanium dioxide is a common 
white pigment, and white latex paints often have high concentrations of titanium in comparison to other trace elements. A study using laser ablation-inductively coupled plasma-mass spectrometry (LA-ICP-MS) demonstrated the concentration of the isotope $\mathrm{Ti}^{48}$ at $\sim 143,000 \mathrm{ppm}$ in Behr Premium Plus 8050 white latex paint, as compared to the next highest concentration element, $\mathrm{Zr}^{90}$, at $\sim 16,800 \mathrm{ppm}$. [6] Other additives may include extenders and plasticizers. Extenders are used to alter properties such as gloss, viscosity, and texture, while plasticizers alter the flexibility of the coating. [3] With the exception of pigments, all of the above mentioned paint components are comprised of organic molecules. This makes instrumental analysis of the organic compounds very attractive, and there are many instrumental techniques currently employed in the forensic analysis of paint.

The most common method of paint analysis is visual inspection, which includes examining the surface for scratches or markings, paint layers (including order and thickness), or the shape of the sample, which could potentially be matched with a sample of known origin. [3] This is a rare occurrence, however, and visual inspection of color can be equally problematic due to human bias. Even instrumental techniques such as microspectrophotometers, which can objectively determine color, are of limited use. This method may be able to determine color, but multiple brands and manufacturers often prepare colors that are nearly identical.

Additional instrumental examination techniques for paint samples include energydispersive x-ray analysis (EDX), Fourier transform infrared microscopy (FTIR), x-ray fluorescence (XRF), and solubility tests. Analysis of paints via EDX has been shown to have the capability to distinguish between brands, but is a qualitative rather than 
quantitative technique. [7] In addition, irregularities in the sample surface can lead to variations in $x$-ray spectral peak intensities as well as atomic number differentiation. [3, 7] Infrared techniques such as FTIR are useful in determining the organic substituents of paint, but these are often a common factor between types of paint (such as latex, lacquer, automotive, etc.) and are therefore more useful in determining the type of paint rather than differentiating between brands or manufacturers. [3] X-ray fluorescence analyses tend to be qualitative in nature. [8] This can be useful for some samples (when overlaying spectra to find matches,) but is problematic for samples that are very similar. Finally, solubility tests have the obvious disadvantage of being destructive to the sample. In addition, many paints can be dissolved by the same solvent, which does not provide any information for discrimination.

Since many of the organic analyses are of limited use, elemental analysis arises as an alternative. Inductively coupled plasma-atomic emission spectroscopy (ICP-AES) has successfully been used to quantitatively determine the concentration of lead in paint, but requires substantial sample preparation such as acid digestions. $[9,10]$ In the case of optical emission spectroscopy (OES), electrothermal vaporization has been used for solid sample introduction of automobile paints. [11] While this significantly decreased the amount of sample preparation, the use of a reactant gas was necessary for analysis. In addition, the method has not been tested on latex paints.

A more extensive examination of white latex paints was reported by Silva et al. [12] However, this study required significant sample preparation, in which paint was dried into powder form in addition to solvent extraction. [12] X-ray diffraction (XRD) was used to determine the crystal structure of the titanium dioxide pigment, while thermal 
gravimetric analysis (TGA) and differential thermal analysis (DTA) were used to identify and quantify resins and pigments. [12] Even with this study, no information was provided as to the discriminating power of the techniques, indicating that the techniques employed were not adequate for forensic applications. Other recent studies of latex paints focus on innovations rather than discrimination. [13-15] Conversely, elemental analyses of paints tend to focus on decorative paints, such as oil paintings [16] and other decorative art (typically of archeological interest.) $[17,18]$ The field of forensic paint analysis, in particular for white architectural paints, is in need of a fast, simple method for elemental analysis and discrimination of paint samples. 


\section{Chapter 2: Background}

The use of laser ablation as a method for solid sample introduction has proven to be quick, reliable, and there is little or no need for sample preparation. Laser ablationinductively coupled plasma-mass spectrometry (LA-ICP-MS) has successfully been used to differentiate 294 out of 300 possible pairs of white latex paint samples. [6] Mass spectrometry is a very sensitive technique, but it does suffer some drawbacks. Mass spectrometers are expensive, difficult to operate, and data interpretation is challenging. A forensic laboratory would need to invest a significant amount of money and time (in the form of training) in order to fully utilize an LA-ICP-MS system.

In contrast, laser induced breakdown spectroscopy (LIBS) is much easier to operate, more affordable, and has simpler data analysis than mass spectrometry. Figure 2.1 illustrates a simple diagram of a LIBS setup. In the LIBS process, a laser (typically either a Nd:YAG or excimer laser) is focused on a small area of the sample. Focusing lenses are used to target the desired position on the sample, and typical spot sizes range from $50-200 \mu \mathrm{m}$. Laser induced breakdown spectroscopy can be performed by ablating multiple spots, but ablation can also be accomplished in rastering mode, in which lines of the sample are ablated. The linear method is often utilized when the area of interest has a shallow depth, in order to avoid ablating undesired portions that may lie beneath the sample. 


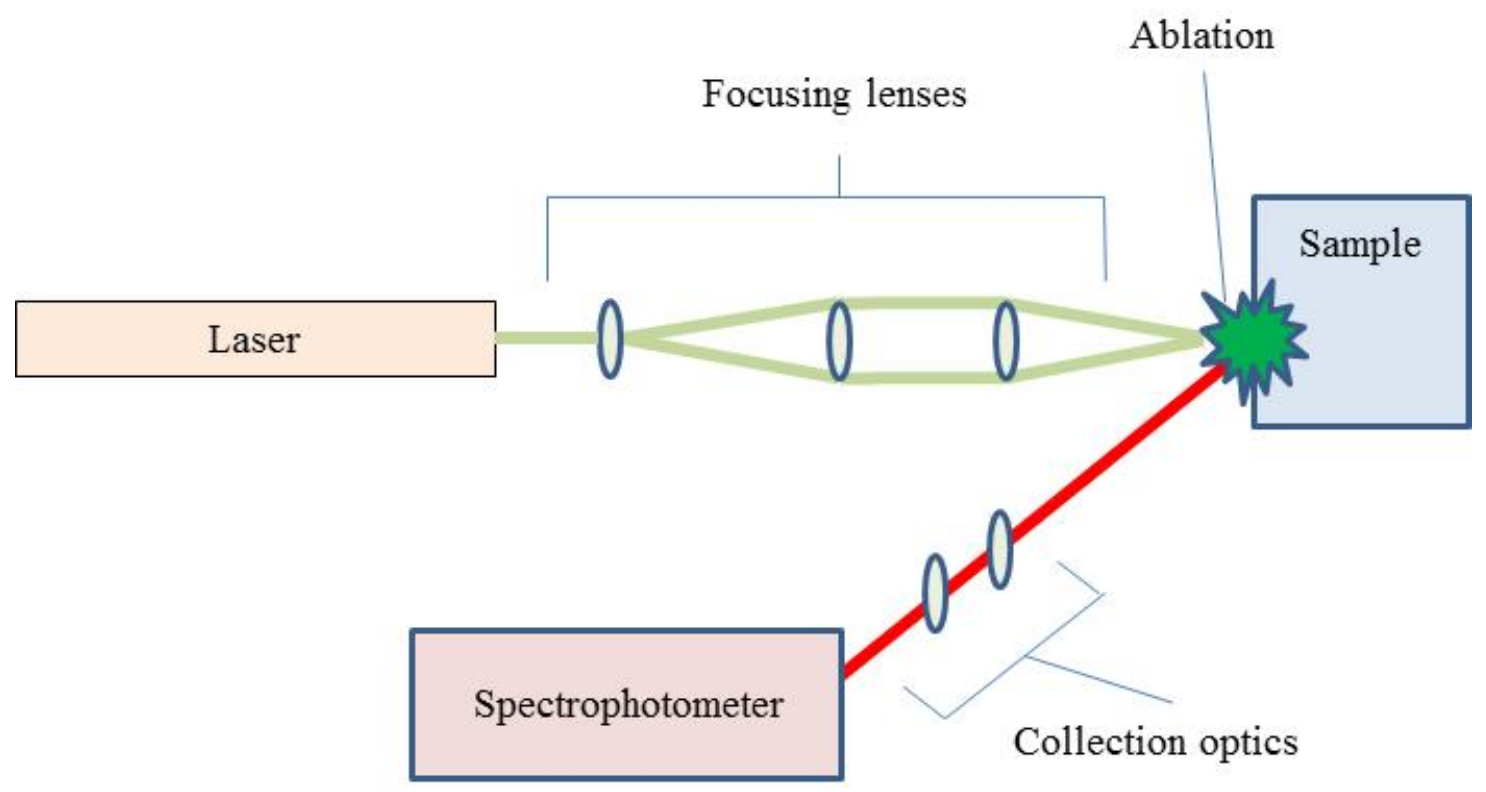

Figure 2.1 A diagram of a LIBS instrumental setup, where a laser is focused onto the sample via focusing lenses. The resulting ablation also creates a plasma, which is assumed to be representative of the sample. As excited species relax to the ground state, a spectrophotometer is used to collect the intensity and wavelength of emission lines.

Once the laser has focused on the sample, ablation occurs and an ionized plasma composed of excited and atomized species is produced. The signal intensity over time is illustrated in Figure 2.2, which demonstrates the signal for a single laser pulse. [19] The gate delay is the amount of time that passes between the initiation of the laser pulse and the beginning of collecting the optical signal via the spectrometer. The gate width, in contrast, is the amount of time the spectrometer measures the signal. These two properties are critical for optimizing LIBS parameters. For example, a gate delay that is too short may result in collection of the laser optical intensity and/or a high level of background continuum radiation. Continuum radiation is a result of white light, bremsstrahlung, and recombination of free electrons and ions in the plasma. [19] As illustrated in Figure 2.2, the high levels of continuum radiation at the early stages of 
plasma formation can severely interfere with the collection of elemental emission lines, particularly those that may have relatively weak intensity. Bremsstrahlung occurs when the kinetic energy of an electron is decreased due to collision with an ion in the plasma, but interferes much less than other contributions to continuum radiation. [20] This illustrates why gate delay must be optimized for LIBS experiments.

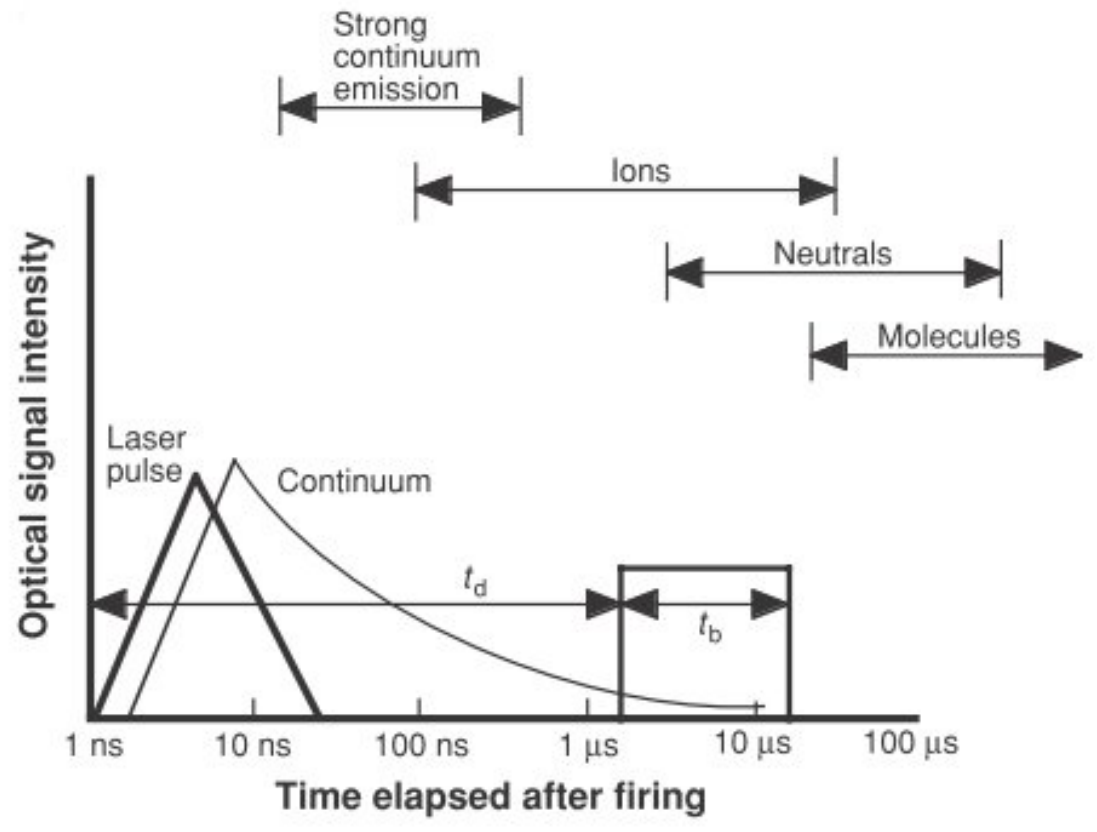

Figure 2.2 The intensity of optical signal decreases over time. [19] The gate delay, $t_{d}$, is the amount of time delay before the spectrometer measures the signal intensity. The gate width, $t_{b}$, is the amount of time the spectrometer measures the emission. Initially, ions are formed due to the formation of the plasma, but recombination of ions and electrons over time results in neutral species being formed. In the final stages of the plasma, molecules reform as the plasma begins to extinguish.

When the excited elements within the plasma relax to the ground state, they emit characteristic photons for which the wavelength and intensity can be recorded with a spectrometer. One of the underlying principles in LIBS is that the plasma is assumed to be representative of the sample, and that a portion of the plasma is in local thermal equilibrium (LTE). [21] Assuming the plasma contains ions and elements in the same 
proportions as the sample, emission lines from these elements can also be expected to be observed in the same proportions (assuming there are no interferences.)The resulting spectra can be used to determine the elemental composition of the sample. Local thermal equilibrium is necessary because the exterior edges of the plasma may be a lower temperature than the center of the plasma, making it unreasonable to assume that the entire plasma is in equilibrium. Local thermal equilibrium focuses on only a portion of the plasma, in which it is more reasonable to assume that the temperature and kinetic energy of that section are in equilibrium.

The gate width, which is the amount of time the spectrometer measures the optical signal, also has a significant impact on the quality of LIBS signal. A long gate width can result in collection of emission after excited species have relaxed, resulting in lower signal intensity. Conversely, a gate width that is too short can also result in low signal intensity and high background due to the short amount of time the emission lines are being monitored. Again, gate width must be optimized in order to maximize signal-tonoise ratios.

Initially, LIBS was viewed as a strictly qualitative technique due to its ability to determine emission lines from specific elements. However, recent advancements have improved to the point where LIBS can be considered at least semi-quantitative. The main obstacles for quantitative analysis in LIBS are matrix effects, such as laser-sample interactions and plasma-particle interactions. One solution to this problem has been the use of matrix-matched standards. By utilizing a matrix of the same composition as the samples, the same matrix effects are expected and therefore accounted for during quantitative analyses. More recent analyses have turned to calibration-free laser ablation 
analyses without matrix matched standards. [22-24] These methods include utilizing a standard aerosol solution mixing with ablated particles [22] as well as mathematical corrections for alterations in laser-matrix interactions. [23] Calibration curves have successfully been acquired via LIBS as well. [25-27] In one case, the concentrations of vanadium and titanium were determined within $4 \%$ of the actual value. [26] Clearly, quantitation of LIBS is continuing to improve with advancing research.

Repeatability and precision has improved for LIBS over time as well. One of the main problems with analysis is the variation in the amount of sample ablated between laser shots. However, this has been combatted with the use of internal standards. [6, 2830] In some cases, an internal standard is selected that is already present in the sample, such as silica for glass. [31] Other methods dope the matrix-matched standard with an internal standard, such as the use of calcium and strontium doped filter papers as calibration standards for water samples. [29] These methods have greatly improved the precision of the LIBS technique.

Laser induced breakdown spectroscopy has successfully been used to determine elemental concentrations in a wide variety of samples, including glass [5, 27], cotton [32], automobile paints [27], paper [4], and ink [4], among others. Utilizing the elemental concentrations, discrimination using LIBS were as follows: $>99 \%$ for glass $[5],>97 \%$ for cotton by US region [32], 97.7\% for printing papers [4], and 96.4\% for black gel inks [4]. These examples demonstrate a broad range of applications for elemental analysis and discrimination via LIBS.

In addition, LIBS has been used to determine the concentrations of lead in household latex paints by using a linear calibration curve. [27] However, this study was 
only applied to two samples of unknown concentration; therefore, the results could not be verified. The study suggests that the concentrations of other elements in latex paints can be quantitatively determined using LIBS, and that these elemental concentrations can be used to discriminate between paint samples.

Laser induced breakdown spectroscopy has the benefit of being nearly nondestructive with little to no required sample preparation. For example, a direct LIBS

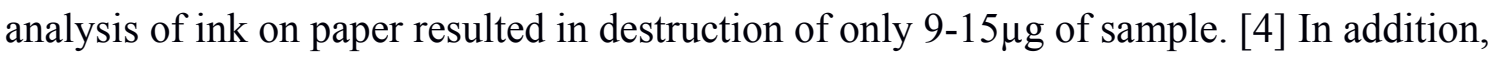
it is low cost, fast, and can be used to analyze multiple elements at the same time.

Despite the advantages of LIBS, there are some discernible drawbacks. Most problematic are the complications that arise from particle-plasma interactions. Plasma formation and growth are strongly related to laser-material interactions, which results in plasma variations in temporal and spatial development, temperature, and free electron density. [33] For example, it is possible for the rate of particle vaporization and rate of dissociation of the sample to be very slow compared to the plasma evolution. This can result in a portion of the analyte remaining bound in a solid particulate, thereby preventing emission and creating a nonlinear analytical response. [34]

A related problem can occur when the rate of heat transfer within the plasma is very slow, causing a local suppression of plasma temperature, electron density, and atomic emission. [34] In addition, continuum background radiation in the early stages of plasma formation interferes with atomic and ionic emission lines. [35]

These problems, however, could be minimized by utilizing a standard addition method to determine the concentration of elements in white latex paints. Because its initial form is liquid, spiking and drying samples is a simple task. In addition, interaction 
between the laser and the sample will be comparable (if not the same) for each standard addition curve, since each standard will be composed of the same paint.

Elemental-based discrimination between white latex paints has been demonstrated previously. A study of 24 white latex paints by Gangon demonstrated $99 \%$ discriminating power using LA-ICP-MS. [6] The comparison method chosen for this analysis was a Tukey's pairwise comparison test. [6] The Tukey method directly compares each elemental signal for every potential pair of paints. For example, the strontium signal at $407 \mathrm{~nm}$ is compared between paint samples A and B. Pairs were considered distinguishable if even one of the elemental pairs could be differentiated between samples. [6] The comparison was repeated for each element of interest for the paint pair; and this process was repeated for every possible paint pair in the sample set. While mass spectroscopy is more sensitive than LIBS, the improvements in precision and quantitation of LIBS suggest that LIBS can also differentiate between samples. 


\section{Chapter 3: Aims of Research}

The main objective of the present research project is to evaluate the utility of LIBS as a tool for the forensic analysis of white latex paints. The process includes determining the discriminating power of LIBS as compared to LA-ICP-MS. Paints analyzed in a previous study by Gagnon [6] will be analyzed in order to compare the discriminating power of each technique. The optimal method of discrimination will be determined, including determining an optimal elemental ratio, the elements used for discrimination, and the best statistical method, such as principle component analysis (PCA) and Tukey's pairwise comparisons. The hypothesis for this research is that, although a different element menu is used for LIBS and LA-ICP-MS, LIBS will discriminate white latex paint samples from different sources with similar power to LAICP-MS. This result is expected because previous analyses utilizing calibration curves with LIBS have been successful, indicating that LIBS is sensitive enough to determine differences in signal intensities. In addition, advances in LIBS research have improved the precision and accuracy of the method.

Although LIBS is still not as precise as LA-ICP-MS, LIBS is expected to be capable of discriminating white latex paints on the basis of elemental signals utilizing an elemental ratio. In addition, LIBS has the benefits of low cost, simple operation, and rapid analysis time, which should prove the method as an attractive model for forensic laboratories. In the forensic laboratory, white latex paints are difficult to distinguish. The most common method is by microscopic observation in an effort to find scratches or other surface markings that will match the sample with a known sample. However, this method is tedious and rarely results in matches. Other techniques, such as FTIR, are only 
useful for determining the type of paint by analyzing the organic components. Typically, the differences in latex, oil based, and other paints can be distinguished visually, making the need for XRF minimal. Methods such as EDX and XRF are qualitative, which is also of limited use. In the case of white latex paints, titanium is expected as one of the main pigments (present as titanium dioxide.) Strictly qualitative analysis provides very little information in this case, since nearly all white latex paint samples contain titanium. In the case of LIBS, the elemental signal increases with an increase in concentration, making it semi-quantitative. Utilizing this advantage, discrimination between white latex paints is made possible. In addition, LIBS is a versatile instrument that can be used for many different samples, such as windshield glass [1], automotive paint [2, 3], paper [4], float glass, and gel ink [5]. The low cost of instrumentation and operation also make it an attractive addition to forensic laboratories, which need to analyze samples quickly. LIBS is a fast technique which requires very little sample- another hallmark of forensic samples. The simplicity of operation and data analysis adds to the rationale of utilizing LIBS in forensic laboratories. 


\section{Chapter 4: Experimental Method Development}

\section{Chapter 4-A: Optimization of LIBS Parameters with White Latex Paint}

\section{Instrumentation}

Analyses were performed on a J200 laser induced breakdown spectroscopy instrument from Applied Spectra. The instrument uses a Nd:YAG laser with a wavelength of $266 \mathrm{~nm}$ as well as an Aurora spectrometer. The peak areas for the lines of interest were determined using Aurora software, including background subtraction and signal-to-noise ratios. Ablated particles were removed from the cell using both argon gas flow and a Sentry Air System HEPA filter. Crater images were acquired with a Keyence digital microscope.

\section{Optimization}

Laser power, gate delay, frequency, gas flow rate, and number of shots per spot were optimized by calculating the signal-to-noise ratio for strontium at the $407 \mathrm{~nm}$ line. A three by three grid (nine spots) of $200 \mu \mathrm{m}$ diameter were used for each optimization, and dry Glidden 2000 (GLD 2000) white latex paint on a glass slide was used as the sample. Argon was used as the carrier gas. The parameters used for each optimization are reported in Table 4.1, and all samples were analyzed on the same day. 
Table 4.1 Optimization parameters for LIBS on Behr 8050 white latex paint are shown below. The parameter being optimized is shown across the top row, while the parameters for each experiment are listed in the corresponding columns.

\begin{tabular}{|c|c|c|c|c|c|}
\hline $\begin{array}{c}\text { Optimized } \\
\text { parameter }\end{array}$ & Laser power & $\begin{array}{c}\text { Gate delay } \\
(\mu \mathrm{s})\end{array}$ & $\begin{array}{c}\text { Frequency } \\
(\mathrm{hz})\end{array}$ & $\begin{array}{c}\text { Gas flow rate } \\
(\mathrm{L} / \mathrm{min})\end{array}$ & $\begin{array}{c}\text { Number of } \\
\text { shots per spot }\end{array}$ \\
\cline { 1 - 5 } $\begin{array}{c}\text { Experimental } \\
\text { parameters }\end{array}$ & - & $80 \%$ & $80 \%$ & $80 \%$ & $80 \%$ \\
\hline Laser power & 1 & - & 1 & 1 & 1 \\
\hline $\begin{array}{c}\text { Gate delay } \\
(\mu \mathrm{s})\end{array}$ & 5 & 5 & - & 5 & 5 \\
\cline { 1 - 4 } $\begin{array}{c}\text { Frequency } \\
(\mathrm{hz})\end{array}$ & 0.6 & 10 & 10 & 50 & - \\
\hline $\begin{array}{c}\text { Gas flow rate } \\
(\mathrm{L} / \mathrm{min})\end{array}$ & 10 & 0.6 & 0.6 & - & 0.6 \\
\hline $\begin{array}{c}\text { Number of } \\
\text { shots per spot }\end{array}$ & & & &
\end{tabular}

The results of optimization are shown in Figures 4.1-4.5 in the form of signal-tonoise ratios. In Figure 4.1, 80\%, 90\%, and 100\% laser power all have similar signal-tonoise ratios. However, $80 \%$ has slightly better precision than the other options and results in a smaller amount of sample being ablated. This could be an important factor if a sample is presented that is not very thick; a deep crater ablation could drill through the entire sample and render the analysis useless. For these reasons, $80 \%$ power was selected as the optimal laser power.

Figure 4.2 indicates the variation in signal-to-noise with alterations in gate delay. The figure clearly shows $0.5 \mu$ s as having the highest signal-to-noise ratio, although it does have higher standard deviation than the lower gate delays of $0.1 \mu$ s and $0.3 \mu$ s. Despite this difference, the gate delay of $0.5 \mu$ s is clearly the best option due to the higher signal-to-noise ratio.

The variation in signal-to-noise ratio with laser shot frequency is shown in Figure 4.3. While there is some overlap between variables when considering the standard 
deviations, 5 hz clearly shows the best signal-to-noise ratio. Similarly, Figure 4.4 shows that $0.6 \mathrm{~L} / \mathrm{min}$ and $1.5 \mathrm{~L} / \mathrm{min}$ both indicate similar signal-to-noise ratios. However, $1.5 \mathrm{~L} / \mathrm{min}$ is a relatively high flow rate and would require a greater amount of argon resources. Therefore, $0.6 \mathrm{~L} / \mathrm{min}$ was selected as the argon flow rate.

The final parameter, shown in Figure 4.5, was optimization of the number of shots taken per spot. While there is relatively little difference between the number of shots examined, 70 shots per spot has both the highest signal-to-noise ratio and, although the signal-to-noise ratio is similar to that of 150 shots, the standard deviation for 70 shots is lower than that of 150 shots. In addition, 150 shots would ablate a larger amount of sample. As mentioned in the determination of optimal laser power, this could be a problem if a sample is presented that is very thin. In conclusion, 70 shots per spot was selected as the best choice for analysis.

Based upon these results, paint analyses were performed at $80 \%$ power, $0.5 \mu \mathrm{s}$ gate delay, $5 \mathrm{hz}, 0.6 \mathrm{~L} / \mathrm{min}$ argon flow, and 70 shots per spot. 


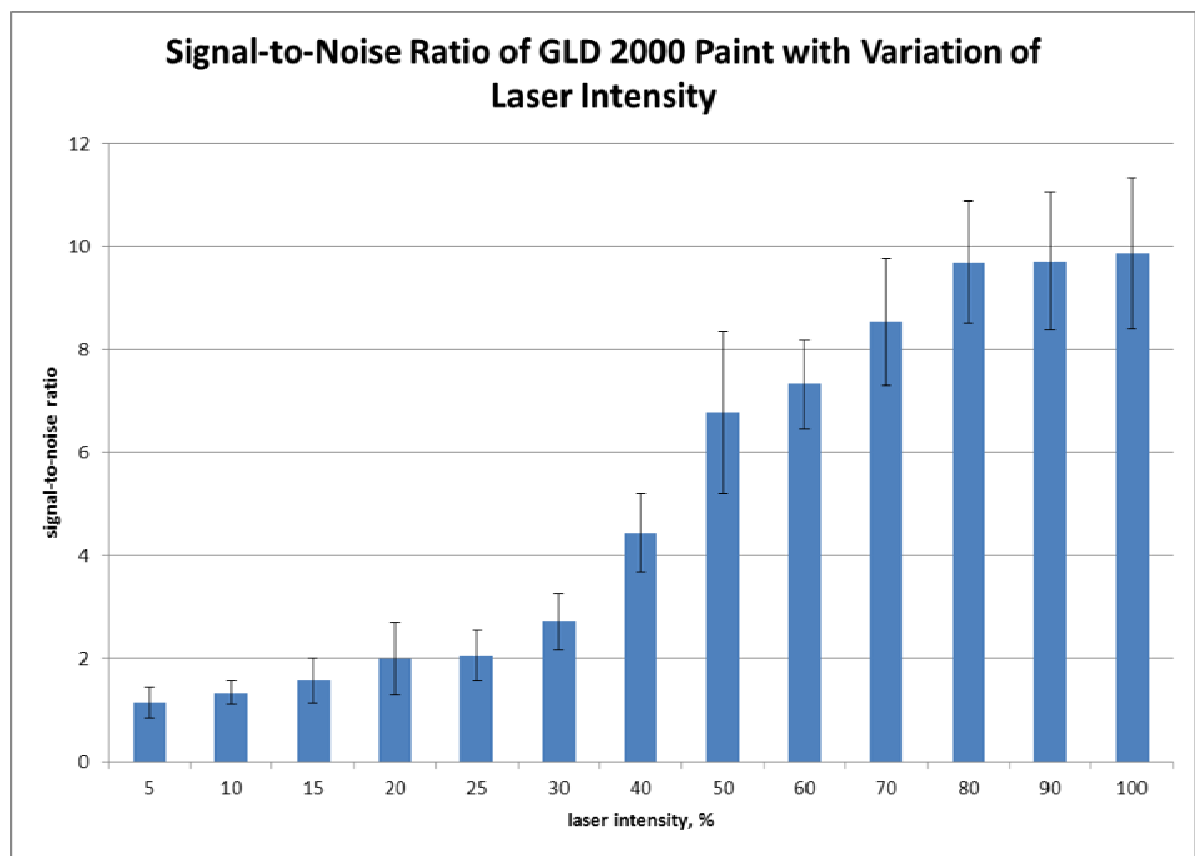

Figure 4.1 Variation in signal-to-noise ratio with change in laser power shows that 80 $100 \%$ intensity gives the best signal-to-noise ratio, with little variation between the three values. 


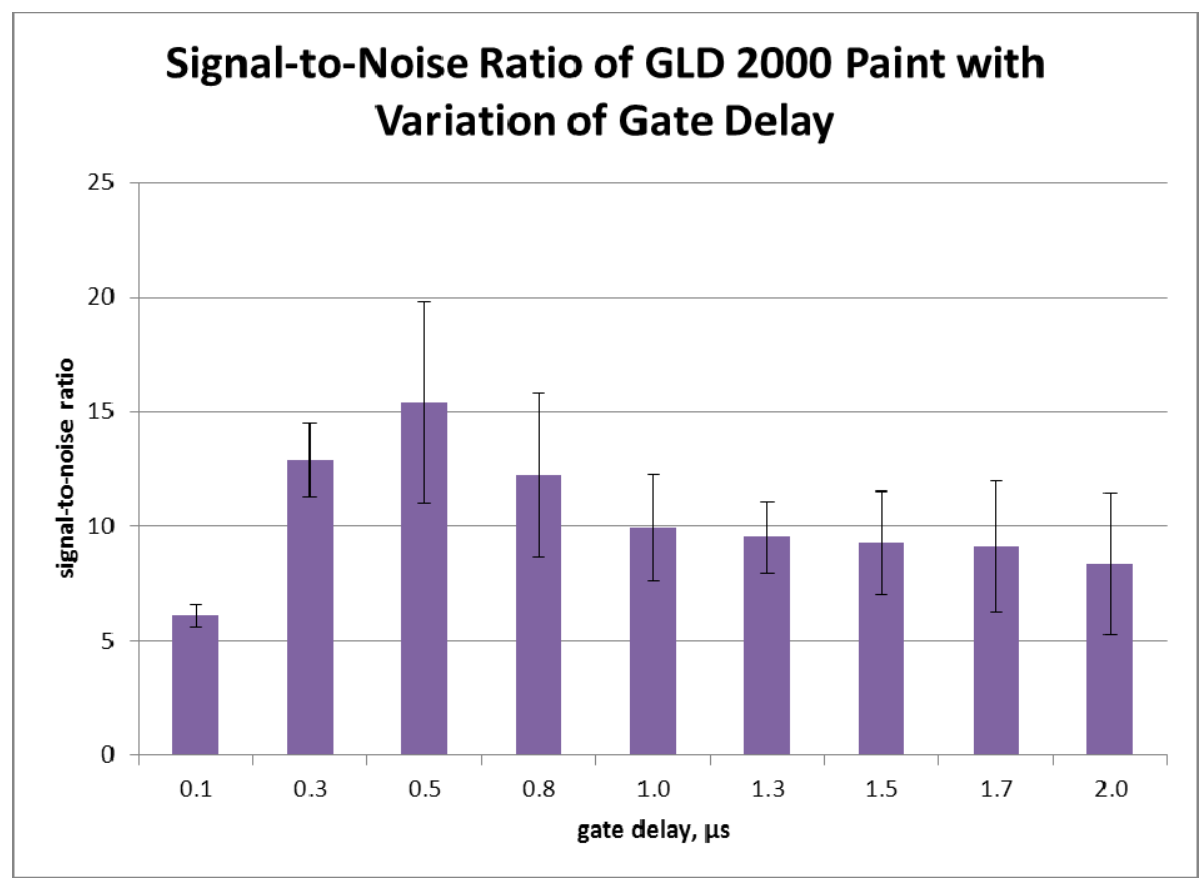

Figure 4.2 Variation in signal-to-noise ratio with change in gate delay shows that the optimal signal-to-noise ratio occurs at $0.5 \mu$ s gate delay.

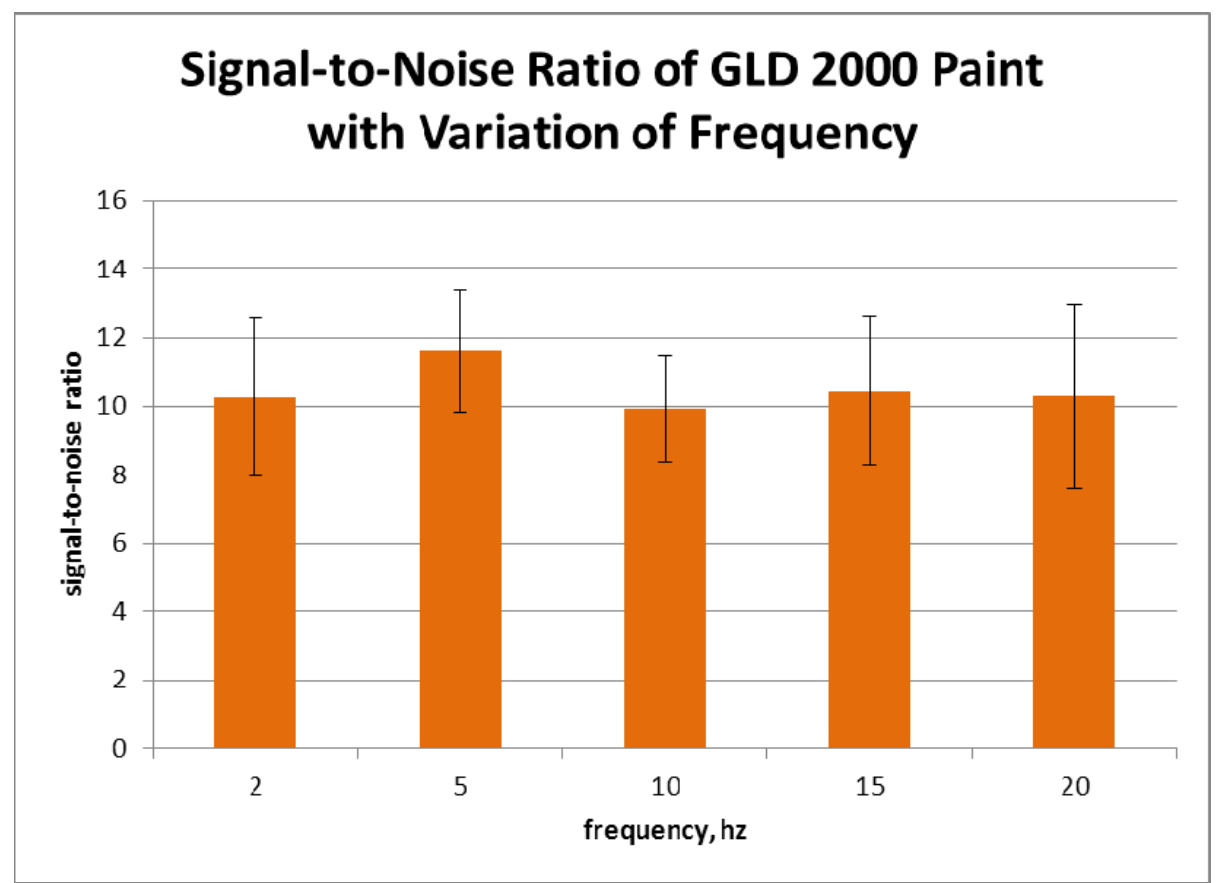

Figure 4.3 Variation in signal-to-noise ratio with change in frequency shows that there is little variation between frequencies when considering the standard deviation of each measurement. However, a frequency of $5 \mathrm{hz}$ appears to show a slightly better signal-tonoise ratio than the other frequencies. 


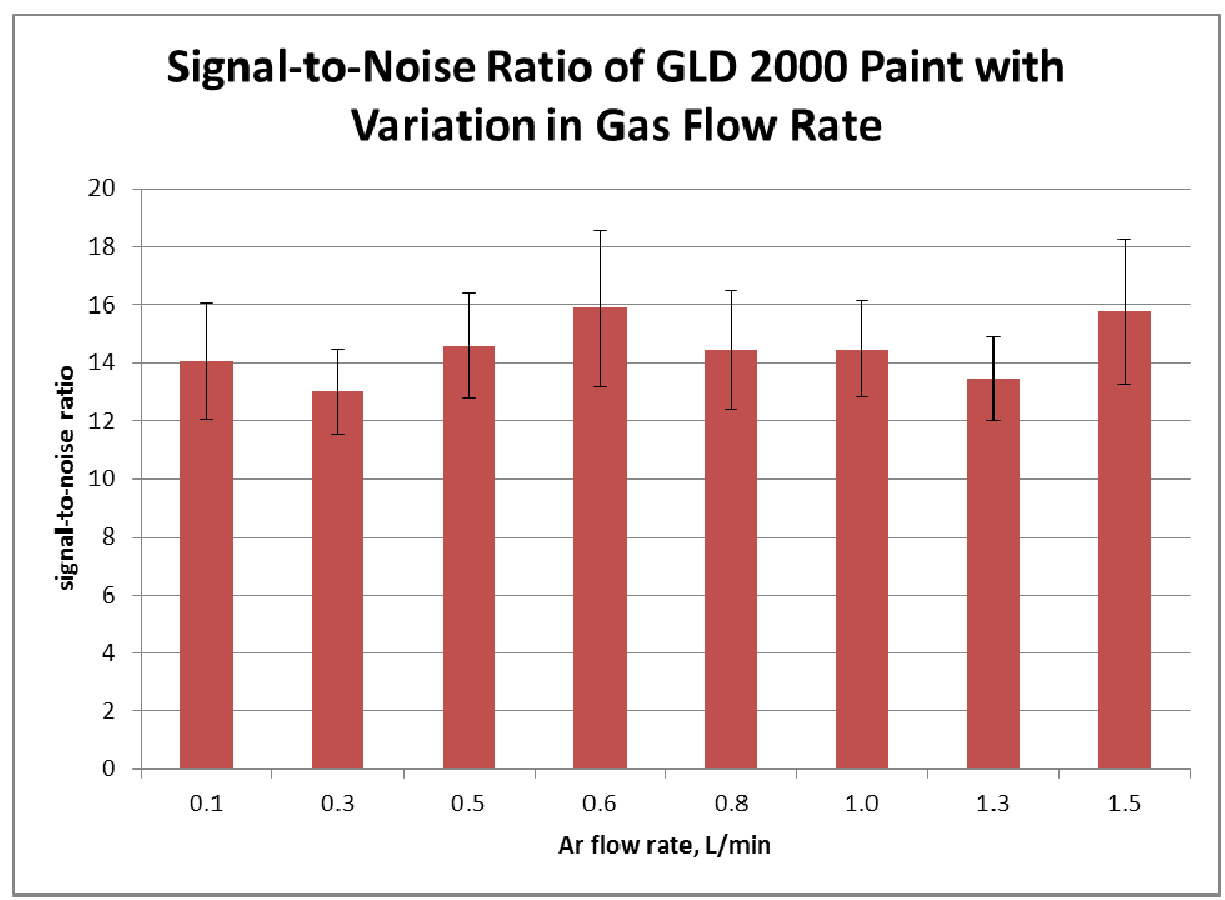

Figure 4.4 Variation in signal-to-noise ratio with change in gas flow rate indicates that $0.6 \mathrm{~L} / \mathrm{min}$ and $1.5 \mathrm{~L} / \mathrm{min}$ demonstrate similar signal-to-noise ratios. The flow rate of $1.5 \mathrm{~L} / \mathrm{min}$, however, is relatively high and would also utilize a higher amount of argon. Therefore, a flow rate of $0.6 \mathrm{~L} / \mathrm{min}$ was selected. 


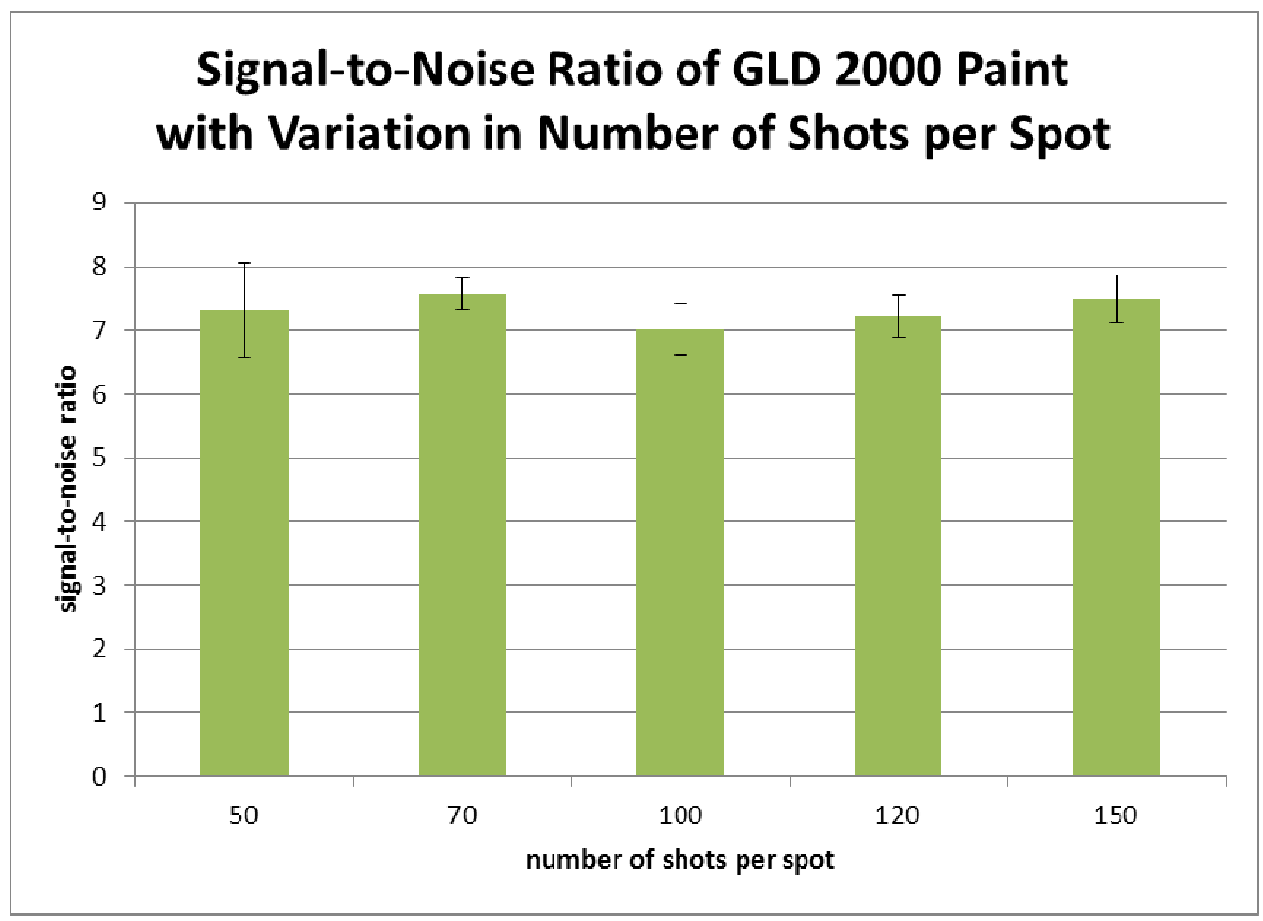

Figure 4.5 Variation in signal-to-noise ratio with change in number of shots per spot shows similar signal-to-noise ratios. The 70 and 150 shots per spot show the best signalto-noise ratios, but 70 shots was selected due to better precision and in order to decrease the amount ablated, which could be important for very thin paint samples.

\section{Chapter 4-B: Previously Analyzed Paint Samples}

The 23 samples from Gagnon [6], as well as one additional sample, GLD2013, were analyzed using a J200 LIBS instrument at the parameters shown in Table 4.2. The GLD2013 sample was acquired within one month of analysis, while the Gagnon samples were prepared in 2006. Sample number 22 was intentionally omitted, as it was not reported by Gagnon. A list of the tested samples is shown in Table 4.3. The element menu and the emission lines analyzed are shown in Table 4.4 and are based on the sample menu used by Gagnon. [6] However, some emission lines could not be differentiated from background, and others suffered from too many interferences to be useful for discrimination. These elements are indicated as such in Table 4.4. 
Laser induced breakdown spectroscopy analysis methods used by Gagnon differed somewhat from the presented research, but these alterations are not expected to have a significant impact on the ability to compare results between LA-ICP-MS and LIBS. For example, Gagnon utilized pelleted samples as well as a linear ablation method instead of multiple spots. The linear method was used due to the determination of a lower mean square as compared to the spot ablations. [6] This method was not utilized in LIBS analysis for several reasons. First, a limited amount of sample was available from Gagnon's previous work, making pelleting unfeasible. In addition, the goal for this experiment is to make a method suitable for forensic laboratories. If differentiation can be determined without the pelleting step, the LIBS differentiation method is more attractive and easier to use than LA-ICP-MS. Although Gagnon used linear ablations for paint analysis, the comparison was between one single spot of $100 \mu \mathrm{m}$ and one linear ablation of the same spot size. [6] Since only one spot was used in Gagnon's comparison, it is not surprising that the linear ablation (which would ablate a larger amount of sample as compared to a single crater,) would produce a lower mean squared error. For the current LIBS experiment, a larger spot size of $200 \mu \mathrm{m}$ was used, in addition to nine different locations on the sample. The large spot size is expected to compensate for any sampling error reported by Gagnon. In addition, a linear ablation would be difficult to accomplish as a result of the uneven surface of the paint samples. Since the samples could not be pelleted due to the small amount available, the paint samples had to be analyzed as they were available- on Teflon backing. The paint had been applied in a dropwise fashion rather than a layered method, resulting in a somewhat uneven sample surface. Utilizing spots in a small area compensated for this by focusing on an area that was relatively flat. 
However, a linear ablation would have required ablation over an uneven surface, which would have introduced additional error to the analyses due to problems with focusing on the sample. The larger spot size $(200 \mu \mathrm{m})$ and multiple spots (nine) should account for the sampling errors reported by Gagnon.

In addition, Gagnon utilized different laser parameters for analysis $(60 \%$ laser power and 5hz.) This is of little concern, however, since Gagnon utilized a Cetac Technologies LSX-200 laser at 266nm, while the LIBS analysis was performed on a J200 instrument specifically designed for both LIBS and laser ablation. The parameters for laser ablation (as in the case of Gagnon) and LIBS are expected to be different, since the laser is performing a different task in each scenario.

As previously indicated, a laser spot size of $200 \mu \mathrm{m}$ was utilized for analysis. Figure 4.6 shows an example of a single crater on a paint sample; this typical crater measures $316 \mu \mathrm{m}$ in diameter at the surface and $189 \mu \mathrm{m}$ at the bottom due to the conical shape. The crater depth is $225 \mu \mathrm{m}$, and the total volume was $9.85 \times 10^{-6} \mathrm{~cm}^{3}$. This is representative of a typical crater resulting from analysis. Since nine craters were made per analysis, the total volume ablated was $8.87 \times 10^{-5} \mathrm{~cm}^{3}$, assuming the same dimensions for all craters. The density of GLD2000, a white latex base paint, was determined by cutting a piece of the dried sample with a razor blade and measuring the water displacement in a $10 \mathrm{~mL}$ graduated cylinder, as well as measuring the mass on a balance. The mass was $0.2886 \mathrm{~g}$, and the volume was $0.13 \mathrm{~mL}$, resulting in an estimated density of $2.2 \mathrm{~g} / \mathrm{cm}^{3}$. Assuming the same density for all paint samples, the total amount ablated per sample is approximately $2.0 \times 10^{-4} \mathrm{~g}$, or $0.20 \mathrm{mg}$. The small amount of mass required for the 
LIBS technique lends itself well to forensic applications, which often require the analysis of small pieces of evidence.

Table 4.2 Paint analysis parameters for the LIBS experiment are indicated below.

\begin{tabular}{|c|c|c|c|c|c|}
\hline $\begin{array}{c}\text { Laser } \\
\text { intensity }\end{array}$ & Gate delay & Frequency & $\begin{array}{c}\text { Argon gas } \\
\text { flow rate }\end{array}$ & $\begin{array}{c}\text { Number of shots } \\
\text { per spot }\end{array}$ & Number of spots \\
\hline $80 \%$ & $0.5 \mu \mathrm{s}$ & $5 \mathrm{hz}$ & $0.6 \mathrm{~L} / \mathrm{min}$ & 70 & $9(3 \mathrm{x} 3$ grid $)$ \\
\hline
\end{tabular}


Table 4.3 A list of examined paint samples from Gagnon [6] are presented below, with the addition of GLD 2013, which was only analyzed via LIBS.

\begin{tabular}{|c|c|c|c|c|c|}
\hline Sample \# & Brand Name & Color & Product \# & Int/Ext & Notes \\
\hline 1 & $\begin{array}{l}\text { Glidden } \\
\text { Evermore }\end{array}$ & Deep tint Base & HG-6980 & Ext & \\
\hline 2 & Ralph Lauren & Brilliant White & RL 1291 & Int & Satin \\
\hline 3 & $\begin{array}{l}\text { Behr Premium } \\
\text { Plus }\end{array}$ & Deep Base & 3300 & Int & Semi-gloss \\
\hline 4 & $\begin{array}{l}\text { Behr Premium } \\
\text { Plus }\end{array}$ & Ultra Pure White & 8050 & Int/Ext & Hi-gloss \\
\hline 5 & $\begin{array}{l}\text { Glidden } \\
\text { Evermore }\end{array}$ & White & HD-6224 & Int & Satin \\
\hline 6 & Quik Hide & White & 26960 & Ext & Flat \\
\hline 7 & Weatherbeater & White & $\underline{30} 37804$ & Ext & Primet \\
\hline 8 & Easy Living & White & $\overline{30} 58754$ & Int/Ext & Primer sealer \\
\hline 9 & ColorPlace & White & 5407 & Int & Semi-gloss \\
\hline 10 & KILZ2 LATEX & White & NONE & Int/Ext & \\
\hline 11 & $\begin{array}{l}\text { McCloskey: } \\
\text { Multiuse }\end{array}$ & White/Light Base & 7445 & Ext & Paint \& primer \\
\hline 12 & $\begin{array}{l}\text { Martha Stewart } \\
\text { Everyday Colors }\end{array}$ & BrightWhite & $24-02$ & Int & Semi-gloss \\
\hline 13 & Dutchboy: Home & Brilliant White & WM.0D7400 & Int & Semi-gloss \\
\hline 14 & $\begin{array}{l}\text { Krylon Color } \\
\text { Creations }\end{array}$ & Gloss White & KDH5001 & Int/Ext & Gloss \\
\hline 15 & $\begin{array}{l}\text { Decotime: } \\
\text { Cabinet Rescue }\end{array}$ & White & DT43 & Int & $\begin{array}{l}\text { Low-luster semi- } \\
\text { matte }\end{array}$ \\
\hline 16 & $\begin{array}{l}\text { Zinsser: } \\
\text { Permawhite }\end{array}$ & Eggshell & 2774 & Int & $\begin{array}{l}\text { Mold/mildew } \\
\text { proof }\end{array}$ \\
\hline 17 & $\begin{array}{l}\text { Rust oleum: } \\
\text { Painter's Touch }\end{array}$ & Gloss White & 1992 & Int/Ext & \\
\hline 18 & $\begin{array}{l}\text { Behr Premium } \\
\text { Plus }\end{array}$ & Ultra Pure White & 1050 & Int & Flat \\
\hline 19 & $\begin{array}{l}\text { Behr Premium } \\
\text { Plus }\end{array}$ & Ultra Pure White & 8050 & Int/Ext & High gloss \\
\hline 20 & $\begin{array}{l}\text { Behr Premium } \\
\text { Plus }\end{array}$ & Pastel Base & 4560 & Ext & Flat \\
\hline 21 & $\begin{array}{l}\text { Martha Stewart: } \\
\text { Everyday Colors }\end{array}$ & IronStone White & $22-40$ & Int & Satin \\
\hline 23 & $\begin{array}{l}\text { Martha Stewart: } \\
\text { Everyday Colors }\end{array}$ & IronStone White & 74-04 & Int & Semi-gloss \\
\hline 24 & $\begin{array}{l}\text { Martha Stewart: } \\
\text { Everyday Colors }\end{array}$ & Magnolia White & $24-06$ & Int & Semi-gloss \\
\hline GLD 2013 & $\begin{array}{l}\text { Glidden Duo } \\
\text { Eggshell Base }\end{array}$ & Eggshell & $2013-16$ & Int & $\begin{array}{l}\text { Base to be tinted } \\
\text { in store }\end{array}$ \\
\hline
\end{tabular}


Table 4.4 The element menu for LIBS analysis is shown below. Notes indicate any prominent interferences, weak intensities, or emission lines that could not be detected.

\begin{tabular}{|c|c|c|}
\hline Element & Wavelength (nm) & Notes \\
\hline $\mathrm{Al}$ & 396.2 & \\
\hline $\mathrm{Ca}$ & 422.8 & \\
\hline $\mathrm{Cr}$ & 520.6 & \\
\hline Co & 252.5 & \\
\hline $\mathrm{Cu}$ & 324.8 & \\
\hline $\mathrm{Fe}$ & 373.7 & Some interference with line at $374.1 \mathrm{~nm}$ \\
\hline $\mathrm{Pb}$ & 280.2 & Interferences with $\mathrm{Au}, \mathrm{Mg}, \mathrm{Zn}$ \\
\hline $\mathrm{Li}$ & 670.8 & \\
\hline $\mathrm{Mg}$ & 516.9 & \\
\hline $\mathrm{Mn}$ & 279.48 & Interference with $\mathrm{Mg} 279.55 \mathrm{~nm}$ \\
\hline $\mathrm{Ni}$ & 243.8 & Interference with Ag 243.8 \\
\hline $\mathrm{K}$ & 766.4 & \\
\hline $\mathrm{Na}$ & 588.0 & Interference from $\mathrm{Ar}$ \\
\hline $\mathrm{Sr}$ & 407.8 & \\
\hline $\mathrm{Zn}$ & 330.3 & \\
\hline $\mathrm{Ta}$ & 265.2 & Weak; interference from Al \\
\hline $\mathrm{Ba}$ & 705.9 & \\
\hline $\mathrm{Rb}$ & 780.0 & \\
\hline $\mathrm{Ag}$ & 243.8 & Some interference from $\mathrm{Ni}$ \\
\hline As & $\mathrm{N} / \mathrm{A}$ & Emission line not detected \\
\hline $\mathrm{Cd}$ & 643.8 & \\
\hline $\mathrm{Si}$ & 390.5 & \\
\hline $\mathrm{Bi}$ & 277.9 & Weak \\
\hline $\mathrm{Ga}$ & 417.2 & Weak \\
\hline $\mathrm{Sc}$ & 363.1 & \\
\hline $\mathrm{Hf}$ & $\mathrm{N} / \mathrm{A}$ & Emission line not detected \\
\hline In & 468.5 & \\
\hline $\mathrm{V}$ & 488.2 & \\
\hline $\mathrm{Sn}$ & $\mathrm{N} / \mathrm{A}$ & Emission line not detected \\
\hline $\mathrm{Ti}$ & 334.9 & \\
\hline $\mathrm{Zr}$ & 361.4 & Some interference from $\mathrm{Sc}$ \\
\hline
\end{tabular}




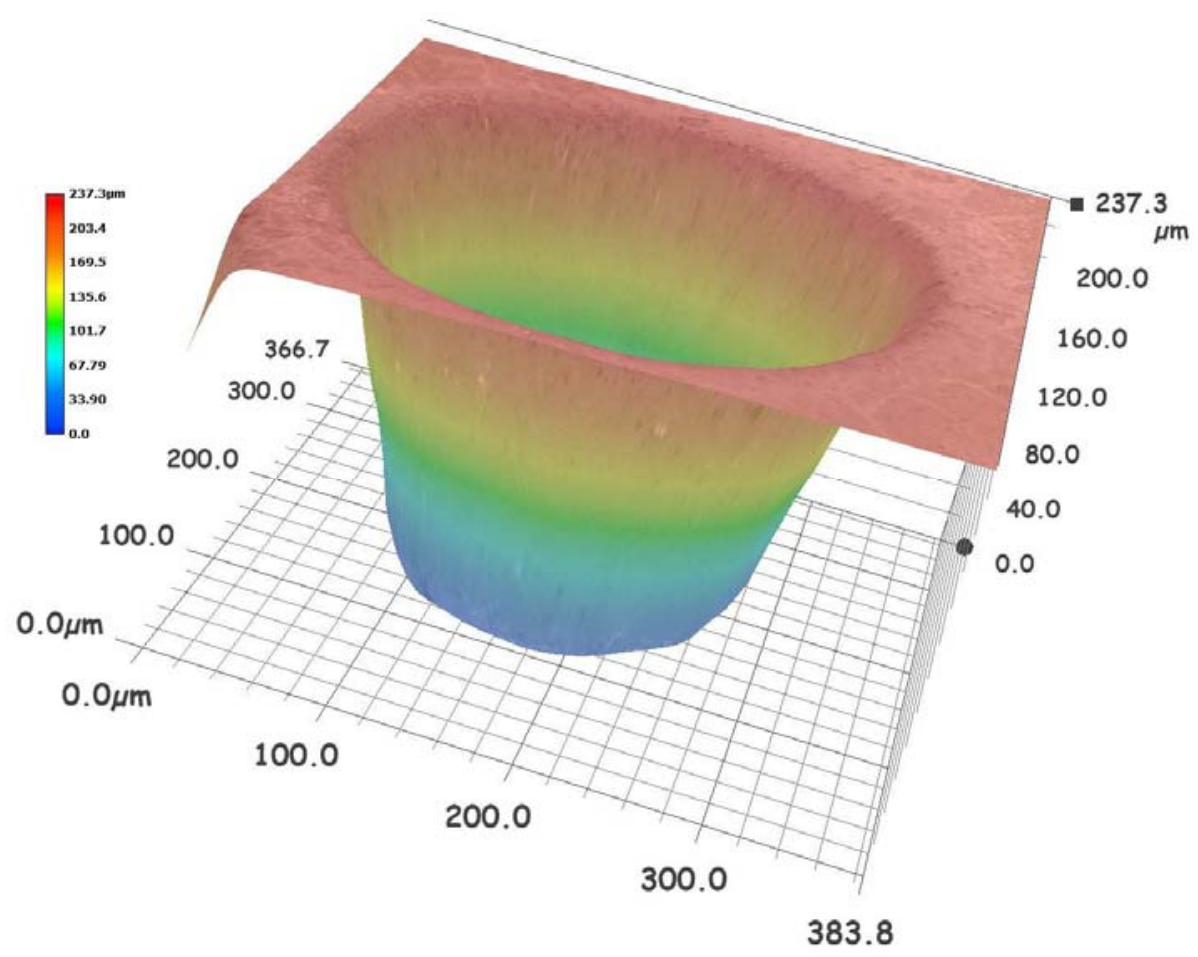

Figure 4.6 A typical crater from LIBS paint analysis is shown above. The conical nature of the crater shape (larger diameter at the top and smaller at the bottom,) is typical of laser ablations.

\section{Chapter 4-C: Determination of LIBS Discrimination Capabilities}

The discriminating power of LIBS was determined using PCA and Tukey's pairwise comparison tests. When analyzing signal intensities, an internal standard or elemental ratio is necessary to account for any variations in the mass of sample ablated, which can vary between laser shots. There were several possibilities for elemental ratios: aluminum, potassium, sodium, and titanium. The selection of the elemental ratio was determined on the basis of relatively high raw intensities seen in each sample compared to other elements, as demonstrated in Figure 4.7. The high intensities in comparison to other elements of interest minimize the impact of variations in the actual concentrations of the elemental ratios between samples. However, sodium was removed as a possibility 
due to high interference with argon at $588.0 \mathrm{~nm}$. As a result, potassium, aluminum, and titanium were all analyzed as potential elemental ratios. 


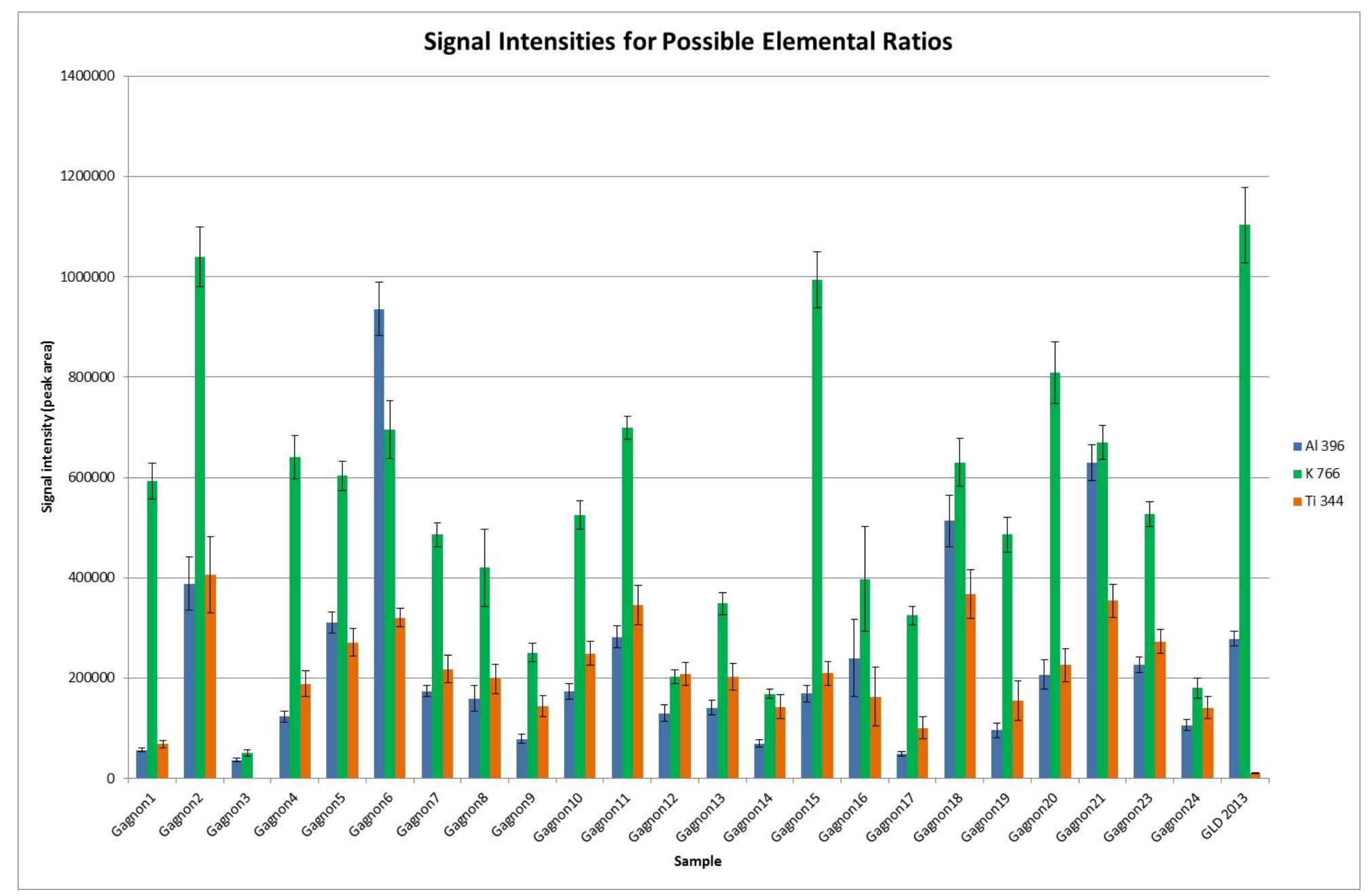

Figure 4.7 Raw intensities of elemental ratio candidates are demonstrated above. Potassium generally shows the highest intensity for each sample, followed by titanium and then aluminum. 
The elements chosen for the statistical tests were determined by examining trends in elemental signal intensity between reports from Gagnon and the LIBS analyzed paint samples. Figure 4.8 shows results from LA-ICP-MS analysis of Behr 8050 as well as results from LIBS analysis of several samples, with other samples omitted for clarity. Each element is normalized to the aluminum signal (for LIBS) or aluminum concentration (for LA-ICP-MS.) The trends in intensity for these elements suggest that they would be good candidates for differentiating white latex paints, since relative increases and decreases are consistent between methods. These consistent trends suggest that LIBS can be expected to demonstrate similar relative sensitivity for these elements. It is important to note, however, that LA-ICP-MS has more overall sensitivity than LIBS. The trends demonstrated in Figure 4.8 simply show the relative response and indicate elements that could be used in differentiation. Elements that did not show increases or decreases consistent with LA-ICP-MS analysis were not considered for the purposes of differentiation. This was, in part, an effort to maintain consistency between LIBS and LA-ICP-MS analysis. Consistent trends for elements between the two methods suggest that both instruments were able to detect the relative signals in a reliable fashion.

The elements utilized for the statistical analyses were barium, copper, strontium, and vanadium, whereas potassium, aluminum, and titanium were examined as elemental ratios. Chromium was omitted because it could not be correctly associated for two different GLD2013 samples (see Table 5.2). For the Tukey test, a $\alpha=0.5$ alpha level was used. If any of the elemental ratios could be differentiated between pairs, the samples were considered discriminant. This is consistent with the differentiation criteria utilized by Gagnon. [6] These calculations were performed using JMP ${ }^{\circledR}$ software. [36] The results 
of the discrimination were compared to the discriminating power of LA-ICP-MS for the same paint samples.

In principle component analysis (PCA), samples are differentiated mathematically by using linear combinations of variables, as shown in Equation 1. In Equation 1, "Z" represents the principle component, " $\mathrm{X}$ " is the variable (in this case, the signal intensity from the elemental lines obtained via LIBS,) and "a" are coefficients that are selected in a manner in which they are not related to each other, unlike the "X" variables. [37] In addition to creating variables that are not correlated, the principle component, " $Z$ ", is chosen such that it accounts for the maximum amount of variation in the data. Multiple principle components can be selected, although typically no more than two or three are utilized. As the number of principle components grow, the amount of variation that the principle component accounts for decreases. The first component will account for the most variation, the second component accounts for the second most variation, and so on. Principle component analysis is an extremely effective method for data reduction, making it an ideal candidate for the LIBS analyses presented here. With five analytes and 23 samples, PCA is an effective tool for determining the amount of variation that can be seen among samples. In addition, PCA can only be performed if there are multiple measurements of the same sample, since differentiation cannot be performed on only one sample. Again, the nine sample spots acquired per sample in the LIBS analysis lends itself well to PCA analysis.

(1) $Z_{1}=a_{11} X_{1}+a_{12} X_{2}+a_{13} X_{3}+\ldots a_{1 n} X_{n}$

In the Tukey's pairwise comparison test, also known as the honestly significant difference (HSD) test, the means of multiple variables are compared. This is a non- 
parametric method, which means that there is no assumption regarding the distribution of the data (such as Gaussian, for example.) [37] In the Tukey's pairwise test, the Studentized range distribution, or $q$ distribution, is used to determine the difference between two means. [38] Equation 2 shows the calculation of the $q$ distribution, where " $\mathrm{n}$ " is the number of measurements, " $\mathrm{T}_{\text {largest" }}$ " and " $\mathrm{T}_{\text {smallest" }}$ are the largest and smallest averages for the sample data, and " $\mathrm{MS}_{\text {error }}$ " is the mean squared error for the experiment. [39] The mean squared error can be acquired from an analysis of variance (ANOVA) test. The resulting $q$ value represents the largest sampling distribution represented in the dataset, and is used for all pairwise comparisons. If the calculated $q$ is larger than the critical value for a given $\alpha$ (the level of significance), then the pairs are considered distinguishable and the null hypothesis is rejected. [38] The critical value for a given number of degrees of freedom can be found on a table of Studentized range distributions.[38]

$$
\text { (2) } q=\frac{\text { Tlargest-Tsmallest }}{\sqrt{\text { MSerror } / \mathrm{n}}}
$$

In this experimental application, a Tukey's pairwise test must be performed for each elemental variable (barium, copper, strontium, and vanadium.) Four Tukey's HSD tests are being performed per elemental ratio. Therefore, each element will result in a different $q$ value, since the largest range in the data will vary between elements (as will the mean square error.) The Tukey's test is ideal for paint differentiation analysis because of the large number of samples being compared, which can be accomplished quickly with mathematical software such as JMP ${ }^{\circledR}$. 


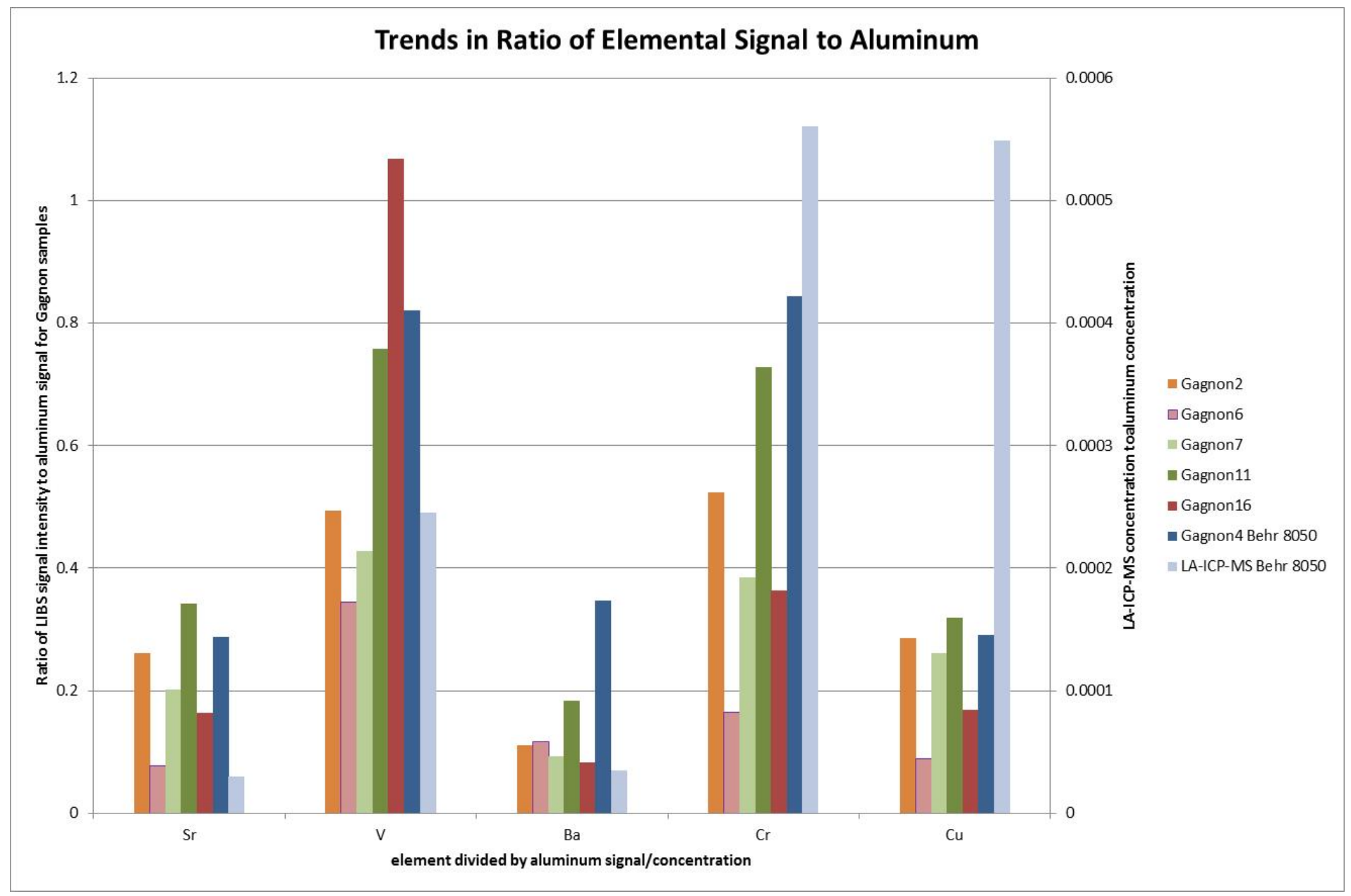

Figure 4.8 Trends in elemental signal between LIBS and LA-ICP-MS data, demonstrated as ratios to aluminum, are shown above. Trends in increases and decreases between elements appear to be consistent between LIBS analysis and LA-ICP-MS analysis by Gagnon. 


\section{Chapter 5: Results and Discussion}

\section{Chapter 5-A: PCA of Gagnon Paint Samples Analyzed via LIBS}

A variety of elemental ratios were examined to determine the best ratios for discrimination. Elemental ratios of aluminum, titanium, and potassium were each examined due to their relatively high signals as compared to other analytes, and the elemental analytes that provided the best discrimination (as described previously)barium, copper, strontium, and vanadium- were utilized for discrimination purposes. Table 5.1 shows the percent discrimination for the first three principle components in each model, as well as which elemental ratios were used.

Table 5.1 The percent account for variation using K, Al, and Ti elemental ratios, respectively, are shown below.

\begin{tabular}{|l|l|l|l|l|}
\hline Ratio used (element variables) & PC1 & PC2 & PC3 & Total \\
\hline Ratio to $\mathrm{K}(\mathrm{Ba}, \mathrm{Cu}, \mathrm{Sr}, \mathrm{V})$ & $75.7 \%$ & $13.5 \%$ & $8.9 \%$ & $98.1 \%$ \\
\hline Ratio to $\mathrm{Al}(\mathrm{Ba}, \mathrm{Cu}, \mathrm{Sr}, \mathrm{V})$ & $73.9 \%$ & $14.7 \%$ & $8.3 \%$ & $96.9 \%$ \\
\hline Ratio to $\mathrm{Ti}(\mathrm{Ba}, \mathrm{Cu}, \mathrm{Sr}, \mathrm{V})$ & $56.4 \%$ & $24.6 \%$ & $13.4 \%$ & $94.4 \%$ \\
\hline
\end{tabular}

The models in Table 5.1 indicate that an elemental ratio of potassium provides the best account of variation at $98.1 \%$ with three principle components, but aluminum also shows a high account for variation at $96.9 \%$. Titanium was anticipated as the best elemental ratio because of the high concentration of the element in white latex paints in the form of titanium dioxide pigment, but resulted in the lowest account of variation for the three possible internals standards (94.4\%). A Tukey's pairwise comparison test to determine correct association between two samples of GLD2013 that were analyzed on 
the same day indicated that most elements could not be correctly associated when titanium was used as an elemental ratio, as shown in Table 5.2. Ideally, all elements would be identified as correctly associated. As a result, titanium was excluded as a possible elemental ratio. Figures 5.1 through 5.4 show the 3D PCA plots for aluminum (Figures 5.1 and 5.2) and potassium (Figures 5.3 and 5.4) as elemental ratios.

Figure 5.1 clearly shows tight clustering for samples such as Gagnon1, Gagnon3, GLD2013blank, Gagnon18, Gagnon7, and Gagnon8, although there appears to be some overlap in the cases of samples 7, 8, and 18. According to the mathematical model, the aluminum elemental ratio accounts for $96.9 \%$ of the variation between samples, which appears to be consistent with the visualization of the 3-D PCA plot. Additional samples can be seen which do not cluster tightly, but do not overlap with other samples, such as Gagnon16, Gagnon17, Gagnon13, and Gagnon14. This suggests that precision for these samples could be improved, possibly by increasing the number of sample shots acquired. However, samples Gagnon4 and Gagnon19 are replicates of the same sample, and indeed show clustering in the same area. In addition, the correct association between the two replicates of GLD2013 (analyzed on the same day) can be seen in Figure 5.2. Both of the aforementioned correctly associated pairs can be seen clustered in the same area, particularly the two samples of GLD2013.

Figure 5.3 demonstrates the 3-D visualization of the PCA analysis for potassium as an elemental ratio. The potassium elemental ratio appears to show tighter clustering for samples than with aluminum as the elemental ratio. This is consistent with the observation that the PCA model with potassium was able to account for $98.1 \%$ of the variation among paint samples, as opposed to the lower value of $96.9 \%$ for aluminum. 
Gagnon14 appears to show the greatest spread and overlaps with samples 6, 12, and 21.The overlap could indicate that discrimination of Gagnon 14 is more difficult than for other samples. Figure 5.4 shows the 3-D PCA plot including the two different samples of GLD2013, where the GLD2013 pair and the Gagnon4 and Gagnon19 pair clusters are indicated with circles. With potassium as the elemental ratio, clustering is still very tight for the two GLD2013 samples. However, there is slightly more overlap with other samples for the Gagnon4 and Gagnon19 samples as opposed to the 3-D PCA plot with aluminum as the elemental ratio. In particular, three of the Gagnon9 measurements appear in close proximity to samples 4 and 19, but the rest of the Gagnon9 measurements can be seen clustered nearby in an area that does not overlap. The variation in Gagnon9 is most likely due to the lower precision of LIBS (as opposed to LA-ICP-MS), and could also be a result of different concentrations in aluminum and potassium in the sample. 
Table 5.2 Tukey pairwise comparison between two GLD 2013 samples, where $\mathrm{x}$ indicates distinguishable elements, is demonstrated below. With titanium as an elemental ratio, only two out of 23 elements could be correctly associated.

\begin{tabular}{|c|c|c|c|c|c|c|c|c|c|c|c|c|c|c|c|c|c|c|c|c|c|c|c|c|c|}
\hline & $\mathrm{Ag}$ & $\mathrm{Al}$ & $\mathrm{Ba}$ & $\mathrm{Bi}$ & $\mathrm{Ca}$ & $\mathrm{Cd}$ & $\mathrm{Co}$ & $\mathrm{Cr}$ & $\mathrm{Cu}$ & $\mathrm{Fe}$ & $\mathrm{In}$ & $\mathrm{Li}$ & $\mathrm{Mg}$ & $\mathrm{Mn}$ & $\mathrm{Ni}$ & $\mathrm{Pb}$ & $\mathrm{Rb}$ & $\mathrm{Sc}$ & $\mathrm{Sr}$ & $\mathrm{Ta}$ & $\mathrm{V}$ & $\mathrm{Zn}$ & $\mathrm{Zr}$ & $\mathrm{Ga}$ & $\mathrm{Si}$ \\
\hline $\begin{array}{c}\text { Raw } \\
\text { intensity }\end{array}$ & & & & & & & & $\mathrm{x}$ & & & & & $\mathrm{x}$ & & & & & & & & & & & $\mathrm{x}$ & \\
\hline Ti ratio & $\mathrm{x}$ & $\mathrm{x}$ & $\mathrm{x}$ & $\mathrm{x}$ & $\mathrm{x}$ & $\mathrm{x}$ & $\mathrm{x}$ & $\mathrm{x}$ & $\mathrm{x}$ & $\mathrm{x}$ & $\mathrm{x}$ & $\mathrm{x}$ & $\mathrm{x}$ & & $\mathrm{x}$ & $\mathrm{x}$ & $\mathrm{x}$ & $\mathrm{x}$ & $\mathrm{x}$ & & $\mathrm{x}$ & $\mathrm{x}$ & $\mathrm{x}$ & $\mathrm{x}$ & $\mathrm{x}$ \\
\hline Al ratio & & - & & & & $\mathrm{x}$ & & $\mathrm{x}$ & & & & $\mathrm{x}$ & $\mathrm{x}$ & & & & & & & & & & & & \\
\hline K ratio & & & & & & $\mathrm{x}$ & & & & & $\mathrm{x}$ & $\mathrm{x}$ & $\mathrm{x}$ & $\mathrm{x}$ & & & & & & & & & & & \\
\hline
\end{tabular}



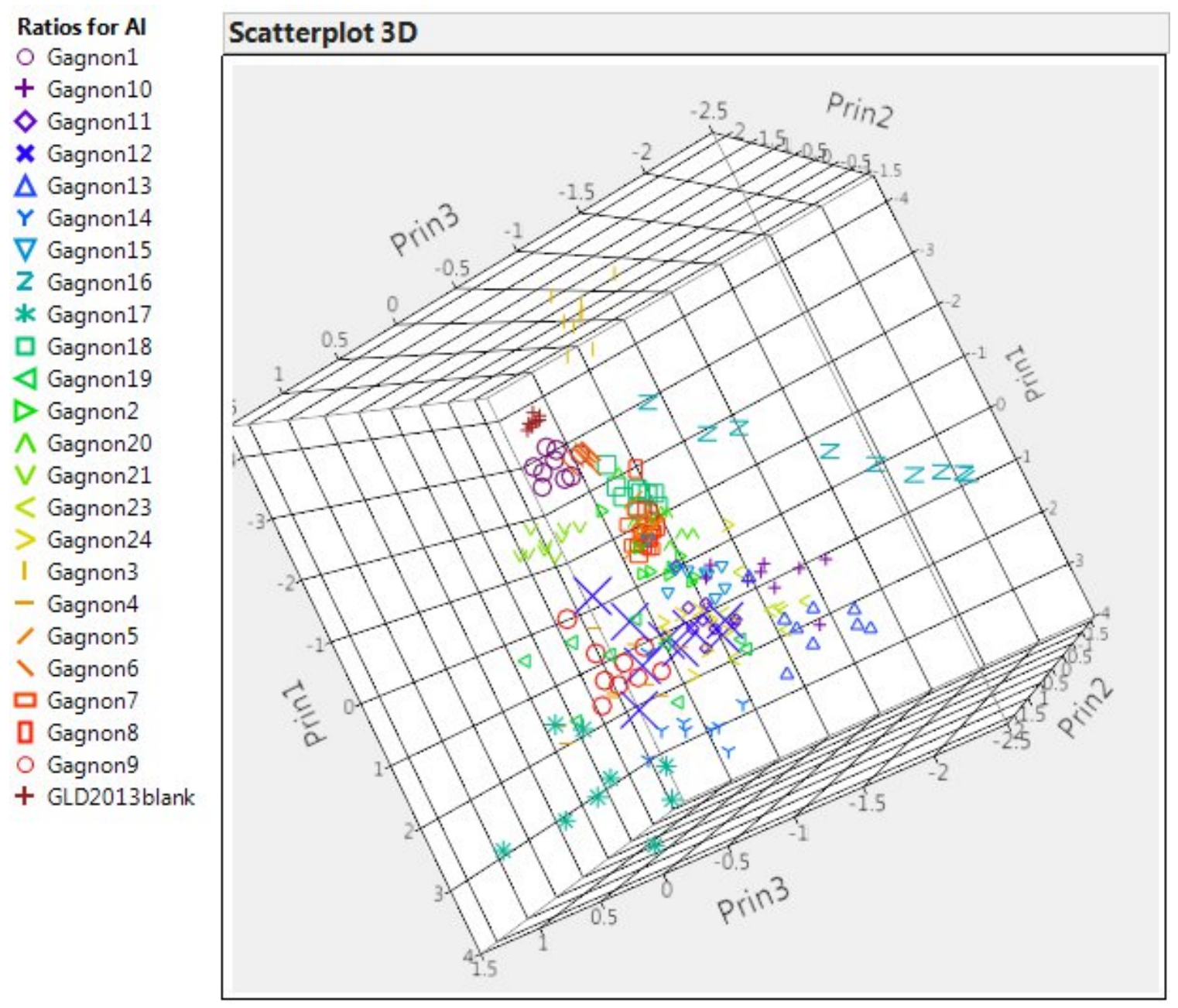

Figure 5.1 A 3-D PCA plot of all paint samples utilizing aluminum as the elemental ratio is shown above. Particularly clear grouping can be seen for Gagnon16, Gagnon1, GLD2013blank, and Gagnon21, among others. 


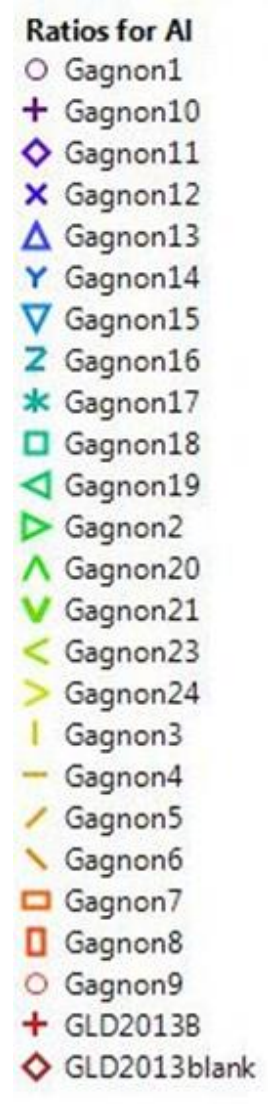

Ratios for Al

- Gagnon1

+ Gagnon10

$\diamond$ Gagnon11

$\times$ Gagnon12

Gagnon13

2 Gagnon 16

* Gagnon17

- Gagnon18

4 Gagnon19

$\triangleright$ Gagnon2

$\wedge$ Gagnon 20

$\checkmark$ Gagnon21

$<$ Gagnon23

$>$ Gagnon24

1 Gagnon3

- Gagnon4

Gagnons

T) Gagnon 8

- Gagnong

+ GLD2013B

GLD2013blank

\section{Scatterplot 3D}

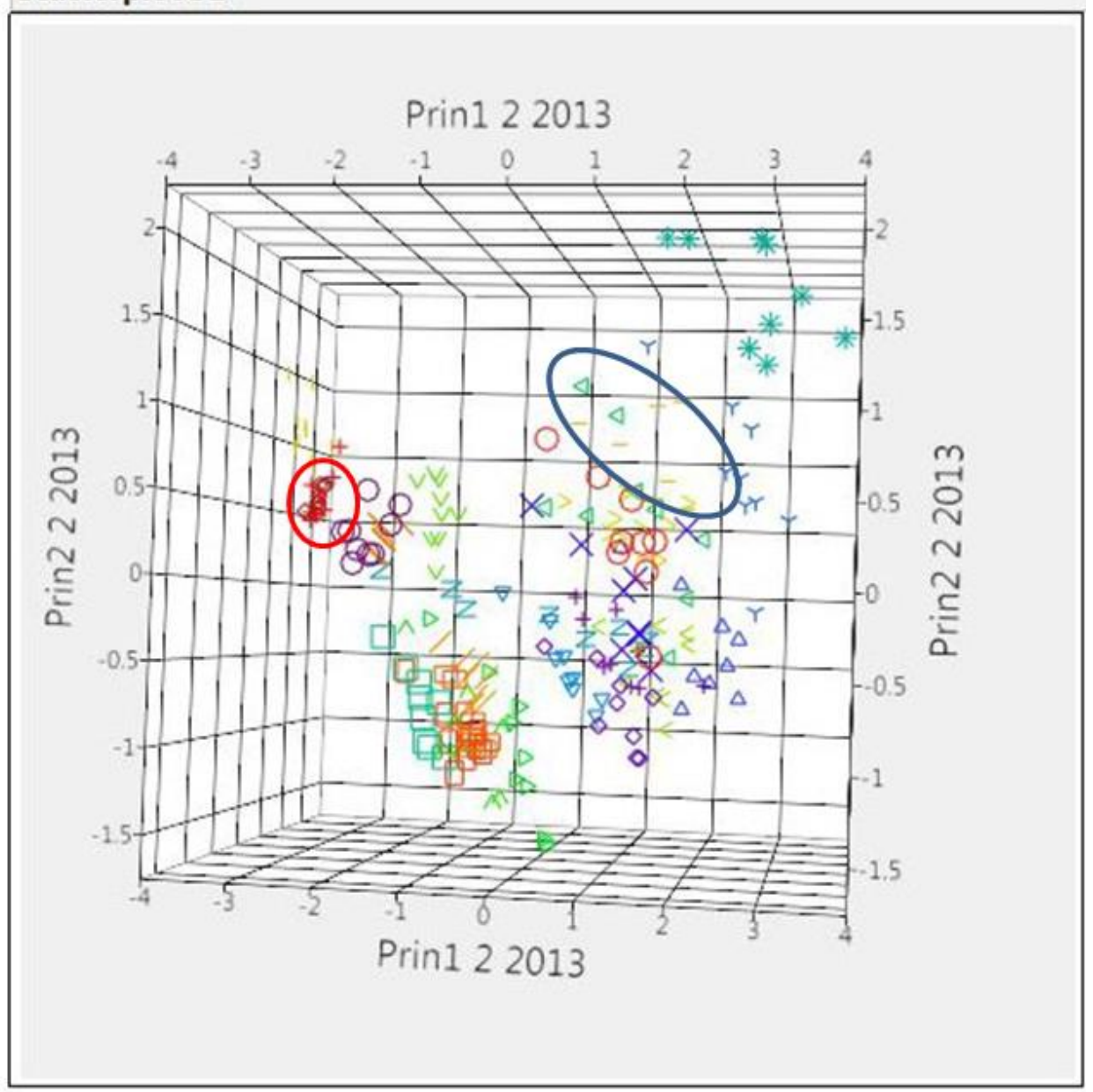

Figure 5.2 A 3-D PCA plot of paint samples including two replicates of GLD2013, utilizing aluminum as the elemental ratio is shown above. Samples Gagnon4 and Gagnon 19 are both the same sample, and show clustering in the same area (as indicated by the blue circled area.) The red circle indicates clustering of the two GLD2013 replicates. 


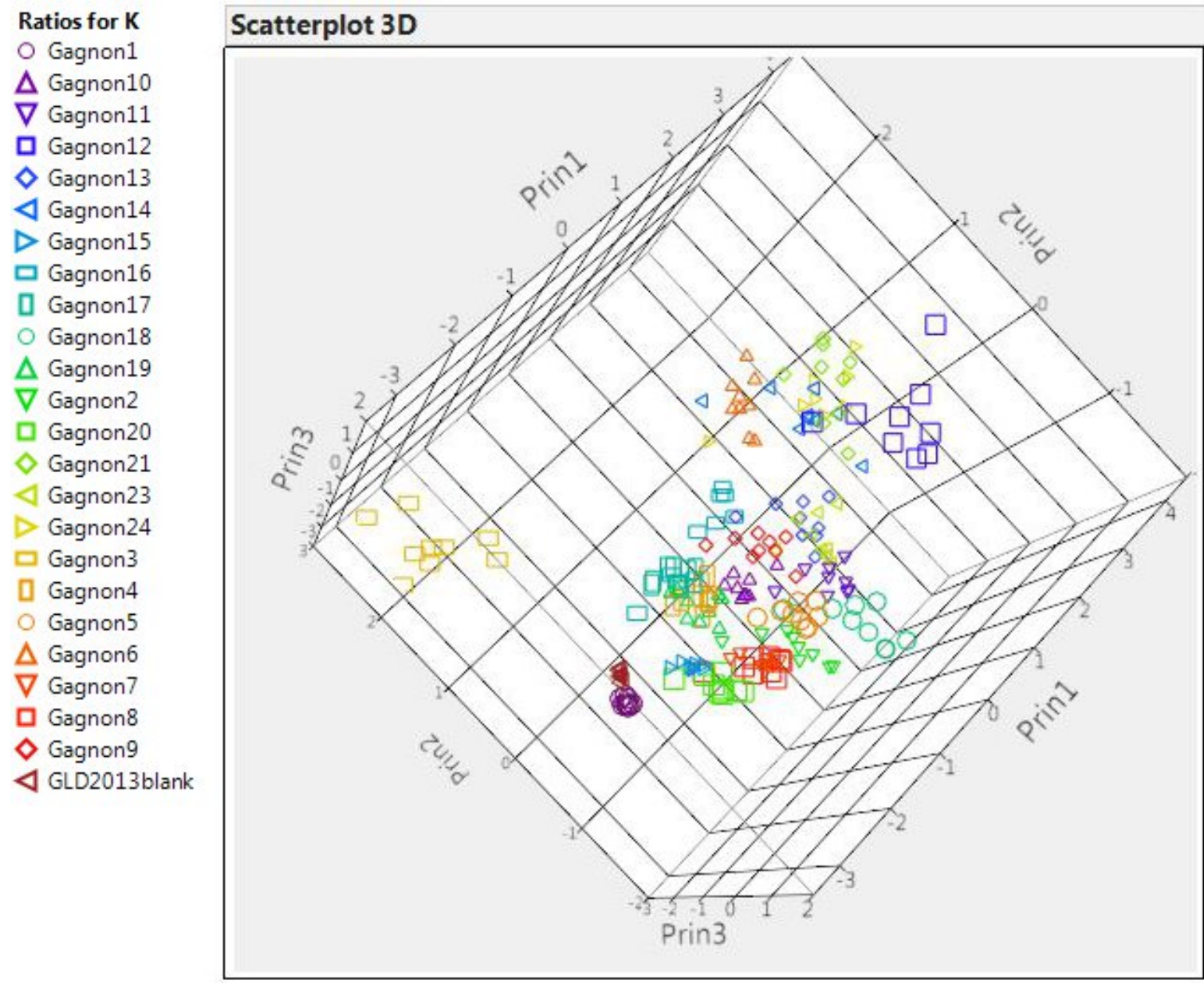

Figure 5.3 A 3-D PCA plot of all paint samples is shown above, with potassium selected as the elemental ratio. Samples Gagnon4 and Gagnon 19 were both the same sample, and show clustering in the same area (as indicated by the circled area.) 

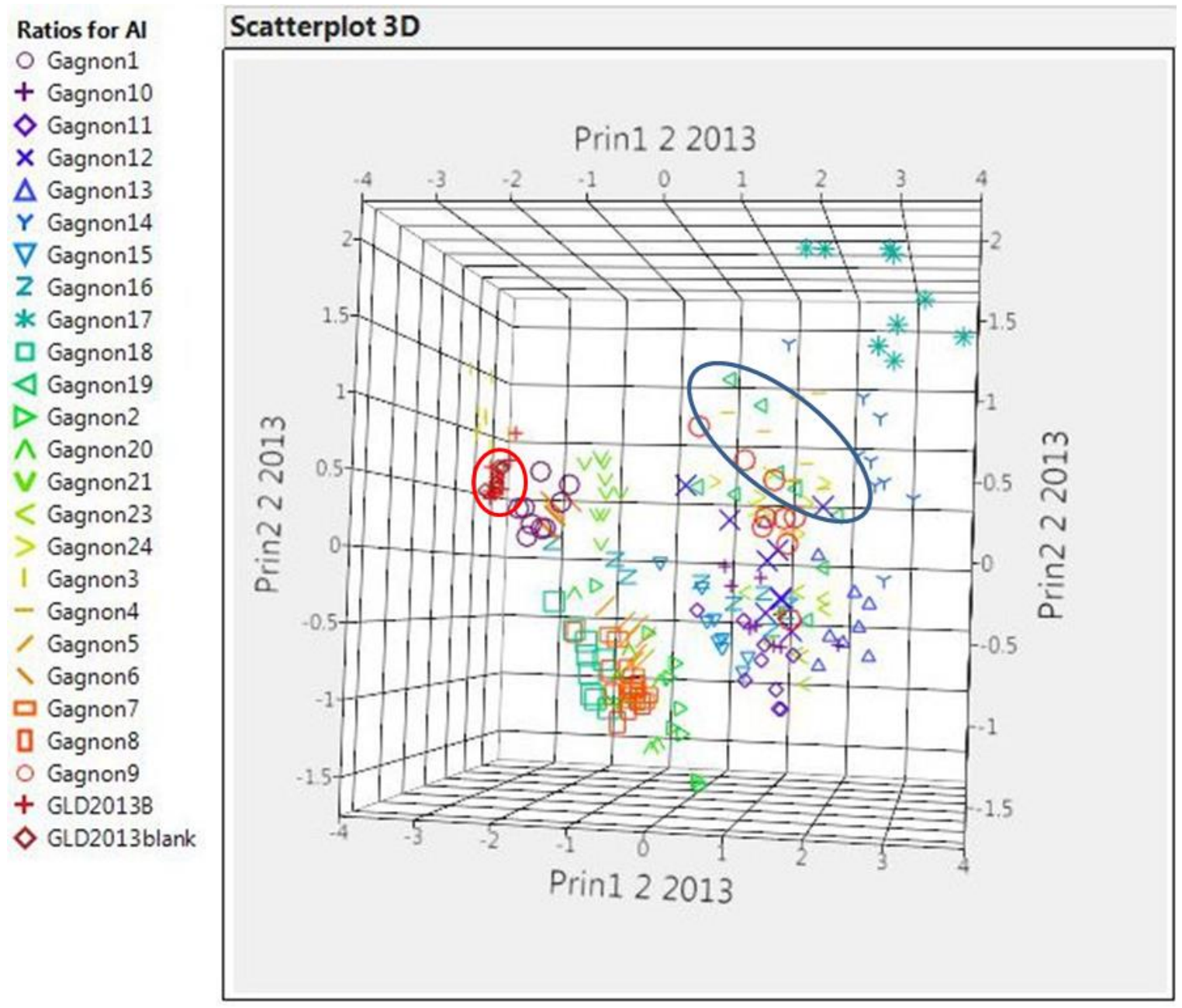

Figure 5.4 A 3-D PCA plot of paint samples including two replicates of GLD2013, utilizing potassium as the elemental ratio is shown above. Samples Gagnon4 and Gagnon 19 are both the same sample, and show clustering in the same area (as indicated by the blue circled area.) However, the clustering seen for potassium as the elemental ratio is not as tight as that seen for aluminum as the elemental ratio. The red circle indicates clustering of the two GLD2013 replicates, which shows tight clustering for both potassium and aluminum as elemental ratios. 
As shown in Figure 5.2, Gagnon 3 (Behr Premium Plus Deep Base) showed clustering furthest from the other samples. Gagnon 3 could be visually differentiated from the other samples as a more yellow color, which could indicate a large difference in elemental makeup. This would be consistent with the observation of greater difference in clustering as compared to other samples.

\section{Chapter 5-B: Tukey's Pairwise Analysis of Gagnon Paint Samples Analyzed by}

\section{LIBS}

The Tukey pairwise discrimination test was applied to 276 possible pairs, of which 264 were distinguishable using potassium as the elemental ratio, and 258 were distinguished using aluminum as the elemental ratio. Tables 5.2 and 5.3 show the pairwise comparisons for potassium and aluminum as elemental ratios, respectively. With potassium as the elemental ratio, 12 pairs could not be discriminated, resulting in $95.6 \%$ discrimination. The elemental ratio of aluminum demonstrated slightly less discrimination, but was still high at $93.5 \%$ discrimination with 18 indistinguishable pairs. Of these pairs, only five were in common between both elemental ratios, listed in Table 5.4. If both methods are utilized, a total discrimination of $98.2 \%$ is achieved. However, analysis with only a single elemental ratio makes the technique much quicker, and therefore more suitable for forensic laboratories. Although the difference in discrimination was small between the two elemental ratios, there was still a difference of $2.1 \%$ discrimination capability. This is most likely the result of the concentration of the elemental ratios. As previously shown in Figure 4.7, the concentrations of potassium in the paint samples were generally higher than that of aluminum. If the concentration of the 
elemental ratio is high (relative to the analytes) throughout all of the samples, any differences in concentration will be relatively small and will have minimal effect on differentiation capabilities. This is consistent with the observation that the potassium elemental ratio provided better discrimination, while the lower concentration aluminum elemental ratio demonstrated lower discriminating power. In addition, the difference in discrimination was small due to the much higher concentration of both aluminum and potassium as compared to the analyte concentrations. Discrimination with aluminum was more affected than potassium because of the lower concentration, but the effect was slight (2.1\%) since the concentration was still relatively high as compared to the analytes.

Sample 7, (Weatherbeater, exterior, primer), was involved in three of the four incorrectly indistinguishable pairs, which could be a result of Gagnon 7's identity as a primer. The purpose of a primer is to create a solid white base upon which other colors can be used and seen more easily. This suggests that the need for pigments other than titanium dioxide (which often contain inorganic metals) is minimized, and these lower concentrations may have been the cause of the three indiscriminant pairs. In addition, one of the pairs (Gagnon 8,) was also a primer, which would be consistent with the above explanation. While fewer elements may have been available in this sample, the low concentrations still allowed for Gagnon 7 to be differentiated in the other 21 possible pairs.

The samples Gagnon 4 and Gagnon 19 were correctly associated utilizing the LIBS technique for both elemental ratios, as they are the same sample from different lots. Figure 5.5 shows the Tukey pairwise comparison for barium with potassium as the elemental ratio. The Figure shows that Gagnon19 and Gagnon4 were indistinguishable 
for barium, and this was also true for copper, strontium, and vanadium. Figure 5.5 also shows distinct differences in barium concentrations for samples $6,12,13,21$, and 24 as compared to the other samples, demonstrating the capability of Tukey's pairwise test to discriminate between samples.

Figure 5.5 A visualization of the Tukey's pairwise comparison test for barium with potassium as an elemental ratio is shown below. Samples Gagnon19 and Gagnon4 are the same sample, and the correct association can be seen below. The samples have been circled for clarity.

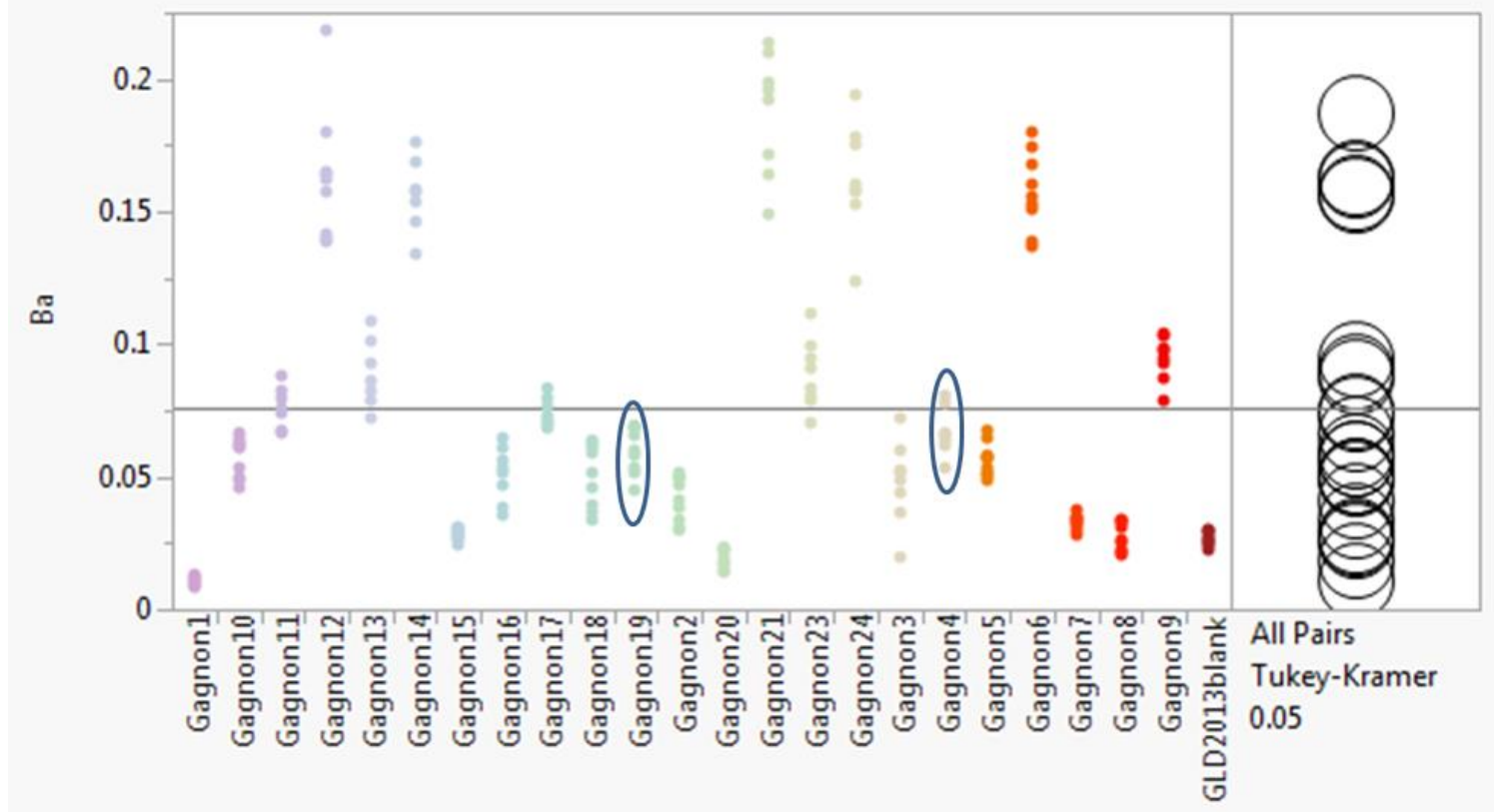

Only four pairs were incorrectly found to be indistinguishable, as shown in Table 5.4. All of the pairs were analyzed using only six elements, (barium, copper, strontium, vanadium, potassium, and aluminum), which may explain why four pairs were indistinguishable. The use of additional elements might result in better discrimination, but would require significantly more analysis time. Considering the low number of elements analyzed and the ease of analysis, $98.2 \%$ discrimination is very efficient. 
The Tukey pairwise comparison results indicate that potassium is better suited as an elemental ratio for white latex paints than aluminum. This is consistent with the results from PCA analysis, where the elemental ratio of potassium was able to account for $98.1 \%$ of the variation amongst samples and the elemental ratio of aluminum accounted for the slightly lower $96.9 \%$. However, utilizing the Tukey pairwise comparison results for both elemental ratios could be useful, as it results in $98.9 \%$ discrimination. The use of both elemental ratios is a strong possibility for forensic laboratories, since all elemental emission signals can be measured during the same analysis. As more elements are added, however, data analysis becomes more time consuming as the intensity of more lines must be examined. Ultimately, the use of potassium as an elemental ratio would be the simplest, fastest, and most effective method for discriminating white latex paints. 
Table 5.2 The pairwise Tukey's test for K as the elemental ratio is shown below, where red indicates pairs that could not be discriminated and green indicates correct association.

\begin{tabular}{|c|c|c|c|c|c|c|c|c|c|c|c|c|c|c|c|c|c|c|c|c|c|c|c|}
\hline & G1 & G10 & G11 & G12 & G13 & G14 & G15 & G16 & G17 & G18 & G19 & G2 & G20 & G21 & G23 & G24 & G3 & G4 & G5 & G6 & G7 & G8 & G9 \\
\hline G10 & 1 & & & & & & & & & & & & & & & & & & & & & & \\
\hline G11 & 1 & 1 & & & & & & & & & & & & & & & & & & & & & \\
\hline G12 & 1 & 1 & 1 & & & & & & & & & & & & & & & & & & & & \\
\hline G13 & 1 & 1 & 1 & 1 & & & & & & & & & & & & & & & & & & & \\
\hline G14 & 1 & 1 & 1 & 1 & 1 & & & & & & & & & & & & & & & & & & \\
\hline G15 & 1 & 1 & 1 & 1 & 1 & 1 & & & & & & & & & & & & & & & & & \\
\hline G16 & 1 & 1 & 1 & 1 & 1 & 1 & 1 & & & & & & & & & & & & & & & & \\
\hline G17 & 1 & 1 & 1 & 1 & 1 & 1 & 1 & 1 & & & & & & & & & & & & & & & \\
\hline G18 & 1 & 1 & 1 & 1 & 1 & 1 & 1 & 1 & 1 & & & & & & & & & & & & & & \\
\hline G19 & 1 & 1 & 1 & 1 & 1 & 1 & 1 & 1 & 0 & 1 & & & & & & & & & & & & & \\
\hline G2 & 1 & 1 & 1 & 1 & 1 & 1 & 1 & 1 & 1 & 1 & 1 & & & & & & & & & & & & \\
\hline G20 & 1 & 1 & 1 & 1 & 1 & 1 & 0 & 1 & 1 & 1 & 1 & 1 & & & & & & & & & & & \\
\hline $\mathrm{G} 21$ & 1 & 1 & 1 & 1 & 1 & 1 & 1 & 1 & 1 & 1 & 1 & 1 & 1 & & & & & & & & & & \\
\hline G23 & 1 & 1 & 1 & 1 & 0 & 1 & 1 & 1 & 1 & 1 & 1 & 1 & 1 & 1 & & & & & & & & & \\
\hline G24 & 1 & 1 & 1 & 1 & 1 & 1 & 1 & 1 & 1 & 1 & 1 & 1 & 1 & 1 & 1 & & & & & & & & \\
\hline G3 & 1 & 1 & 1 & 1 & 1 & 1 & 1 & 1 & 1 & 1 & 1 & 1 & 1 & 1 & 1 & 1 & & & & & & & \\
\hline G4 & 1 & 1 & 1 & 1 & 1 & 1 & 1 & 1 & 0 & 1 & 0 & 1 & 1 & 1 & 1 & 1 & 1 & & & & & & \\
\hline G5 & 1 & 0 & 0 & 1 & 1 & 1 & 1 & 1 & 1 & 1 & 1 & 0 & 1 & 1 & 1 & 1 & 1 & 1 & & & & & \\
\hline G6 & 1 & 1 & 1 & 1 & 1 & 1 & 1 & 1 & 1 & 1 & 1 & 1 & 1 & 1 & 1 & 1 & 1 & 1 & 1 & & & & \\
\hline G7 & 1 & 1 & 1 & 1 & 1 & 1 & 1 & 1 & 1 & 1 & 1 & 0 & 0 & 1 & 1 & 1 & 1 & 1 & 1 & 1 & & & \\
\hline G8 & 1 & 1 & 1 & 1 & 1 & 1 & 1 & 1 & 1 & 1 & 1 & 1 & 1 & 1 & 1 & 1 & 1 & 1 & 1 & 1 & 0 & & \\
\hline G9 & 1 & 1 & 1 & 1 & 1 & 1 & 1 & 1 & 1 & 1 & 1 & 1 & 1 & 1 & 1 & 1 & 1 & 1 & 1 & 1 & 1 & 1 & \\
\hline GLD & 0 & 1 & 1 & 1 & 1 & 1 & 1 & 1 & 1 & 1 & 1 & 1 & 1 & 1 & 1 & 1 & 1 & 1 & 1 & 1 & 1 & 1 & 1 \\
\hline
\end{tabular}


Table 5.3 The pairwise Tukey's test for Al as the elemental ratio is shown below, where red indicates pairs that could not be discriminated and green indicates correct association.

\begin{tabular}{|c|c|c|c|c|c|c|c|c|c|c|c|c|c|c|c|c|c|c|c|c|c|c|c|}
\hline & G1 & G10 & G11 & G12 & G13 & G14 & G15 & G16 & G17 & G18 & G19 & G2 & G20 & G21 & G23 & G24 & G3 & G4 & G5 & G6 & G7 & G8 & G9 \\
\hline G10 & 1 & & & & & & & & & & & & & & & & & & & & & & \\
\hline G11 & 1 & 1 & & & & & & & & & & & & & & & & & & & & & \\
\hline G12 & 1 & 1 & 1 & & & & & & & & & & & & & & & & & & & & \\
\hline G13 & 1 & 1 & 1 & 1 & & & & & & & & & & & & & & & & & & & \\
\hline G14 & 1 & 1 & 1 & 1 & 1 & & & & & & & & & & & & & & & & & & \\
\hline G15 & 1 & 1 & 0 & 1 & 1 & 1 & & & & & & & & & & & & & & & & & \\
\hline G16 & 1 & 1 & 1 & 1 & 1 & 1 & 1 & & & & & & & & & & & & & & & & \\
\hline G17 & 1 & 1 & 1 & 1 & 1 & 1 & 1 & 1 & & & & & & & & & & & & & & & \\
\hline G18 & 1 & 1 & 1 & 1 & 1 & 1 & 1 & 1 & 1 & & & & & & & & & & & & & & \\
\hline G19 & 1 & 1 & 1 & 1 & 1 & 1 & 1 & 1 & 1 & 1 & & & & & & & & & & & & & \\
\hline $\mathrm{G} 2$ & 1 & 1 & 1 & 1 & 1 & 1 & 1 & 1 & 1 & 1 & 1 & & & & & & & & & & & & \\
\hline $\mathrm{G} 20$ & 1 & 1 & 1 & 1 & 1 & 1 & 1 & 1 & 1 & 0 & 1 & 0 & & & & & & & & & & & \\
\hline $\mathrm{G} 21$ & 1 & 1 & 1 & 1 & 1 & 1 & 1 & 1 & 1 & 1 & 1 & 1 & 1 & & & & & & & & & & \\
\hline $\mathrm{G} 23$ & 1 & 1 & 1 & 1 & 1 & 1 & 1 & 1 & 1 & 1 & 1 & 1 & 1 & 1 & & & & & & & & & \\
\hline G24 & 1 & 1 & 1 & 0 & 1 & 1 & 1 & 1 & 1 & 1 & 0 & 1 & 1 & 1 & 1 & & & & & & & & \\
\hline G3 & 1 & 1 & 1 & 1 & 1 & 1 & 1 & 1 & 1 & 1 & 1 & 1 & 1 & 1 & 1 & 1 & & & & & & & \\
\hline G4 & 1 & 1 & 1 & 1 & 1 & 1 & 1 & 1 & 1 & 1 & 0 & 1 & 1 & 1 & 1 & 1 & 1 & & & & & & \\
\hline G5 & 1 & 1 & 1 & 1 & 1 & 1 & 1 & 1 & 1 & 1 & 1 & 0 & 0 & 1 & 1 & 1 & 1 & 1 & & & & & \\
\hline G6 & 1 & 1 & 1 & 1 & 1 & 1 & 1 & 1 & 1 & 1 & 1 & 1 & 1 & 1 & 1 & 1 & 1 & 1 & 1 & & & & \\
\hline G7 & 1 & 1 & 1 & 1 & 1 & 1 & 1 & 1 & 1 & 0 & 1 & 0 & 0 & 1 & 1 & 1 & 1 & 1 & 0 & 1 & & & \\
\hline G8 & 1 & 1 & 1 & 1 & 1 & 1 & 1 & 1 & 1 & 0 & 1 & 1 & 0 & 1 & 1 & 1 & 1 & 1 & 0 & 1 & 0 & & \\
\hline G9 & 1 & 1 & 1 & 1 & 1 & 1 & 1 & 1 & 1 & 1 & 0 & 1 & 1 & 1 & 1 & 1 & 1 & 1 & 1 & 1 & 1 & 1 & \\
\hline GLD & 1 & 1 & 1 & 1 & 1 & 1 & 1 & 1 & 1 & 1 & 1 & 1 & 1 & 1 & 1 & 1 & 1 & 1 & 1 & 0 & 1 & 1 & 1 \\
\hline
\end{tabular}


Table 5.4 Pairs that could not be distinguished via Tukey's pairwise analysis are indicated below.

\begin{tabular}{|l|l|}
\hline Gagnon 4 \& Gagnon 19 & $\begin{array}{l}\text { Both are Behr Premium Plus, Ultra Pure White, } \\
\text { interior/exterior, high gloss (correct association) }\end{array}$ \\
\hline Gagnon 2 \& Gagnon 5 & $\begin{array}{l}\text { Ralph Lauren, Brilliant White, interior, satin \& Quik Hide, } \\
\text { exterior, flat }\end{array}$ \\
\hline Gagnon 2 \& Gagnon 7 & $\begin{array}{l}\text { Ralph Lauren, Brilliant White, interior, satin \& } \\
\text { Weatherbeater, exterior, primer }\end{array}$ \\
\hline Gagnon 7 \& Gagnon 8 & $\begin{array}{l}\text { Weatherbeater, exterior, primer \& Easy Living, } \\
\text { Interior/exterior, primer/sealer }\end{array}$ \\
\hline Gagnon 7 \& Gagnon 20 & $\begin{array}{l}\text { Weatherbeater, exterior, primer \& Behr Premium Plus, pastel } \\
\text { base, flat }\end{array}$ \\
\hline
\end{tabular}

\section{Chapter 5-C: Comparison of Discrimination Power Between LIBS and LA-ICP-MS}

Laser induced breakdown spectroscopy has been demonstrated as an effective tool for discriminating white latex paints. However, the discriminating capabilities were not as effective as those of LA-ICP-MS. Using the same sample set, LA-ICP-MS resulted in 99\% discrimination power via the Tukey pairwise comparison test [6], whereas LIBS showed 95.6\% discrimination with Tukey's pairwise comparison and PCA was able to account for $98.1 \%$ of variation (with potassium as the elemental ratio.) Since mass spectrometry is more sensitive, it was expected that LA-ICP-MS would result in better discriminating power than LIBS. However, it should be noted that 32 elemental isotopes were used for mass spectrometric analysis, [6] whereas only five elements were necessary for LIBS discrimination (six if comparing results for both potassium and aluminum as elemental ratios.) These results indicate that the data analysis process for LIBS is much less complex than for LA-ICP-MS, which can improve efficiency for forensic laboratory analyses.

In addition, LIBS was able to discriminate between the three pairs that could not be discriminated via LA-ICP-MS using Tukey's pairwise analysis. These pairs are shown 
in Table 5.5. These results are surprising, since mass spectrometry is more sensitive than LIBS. The expected outcome was that LIBS would be unable to distinguish the same pairs as LA-ICP-MS, as well as some additional pairs that could be discriminated with mass spectrometry. These results could be due to the elemental ratio selected by Gagnon, which was silica, not potassium. Silica was not selected as a possible elemental ratio for LIBS experiments because it showed much lower intensity than aluminum and potassium. For example, the ratio of aluminum to silica for GLD2013 was 4.64, and the ratio of potassium to silica was even higher at 18.4. These differences in intensity suggested that silica would not be a suitable candidate as an elemental ratio during LIBS analysis and may account for the difference in indistinguishable pairs between LIBS and LA-ICP-MS.

Table 5.5 Indistinguishable pairs reported by Gagnon [6] are shown below; only 3 pairs were indistinguishable.

\begin{tabular}{|l|l|}
\hline Gagnon 15 \& Gagnon 20 & $\begin{array}{l}\text { Decotime: Cabinet Rescue, interior, low-luster semi-matte \& } \\
\text { Behr Premium Plus, pastel base, flat }\end{array}$ \\
\hline Gagnon 23 \& Gagnon 9 & $\begin{array}{l}\text { Martha Stewart: Everyday Colors, Ironstone White, interior, } \\
\text { semi-gloss \&Colorplace, interior, semi-gloss }\end{array}$ \\
\hline Gagnon 20 \& Gagnon 21 & $\begin{array}{l}\text { Behr Premium Plus, pastel base, flat \& Martha Stewart: } \\
\text { Martha Stewart: Everyday Colors, Ironstone White, interior, } \\
\text { satin }\end{array}$ \\
\hline
\end{tabular}

In PCA analysis, LIBS performed well and accounted for $98.1 \%$ of variation between samples. This is comparable to the $99 \%$ discrimination demonstrated by LAICP-MS; however, discrimination should not be confused with the ability to account for variation. The PCA analyses indicate a strong probability that the white latex paints samples could be differentiated, which was substantiated by the Tukey's pairwise comparison tests. In addition to good discrimination capabilities, LIBS analysis is faster, 
simpler, and the instrumentation less expensive than LA-ICP-MS. Again, considering the aforementioned benefits of LIBS, as well as the comparable discriminating capability, LIBS should be considered a valuable alternative to LA-ICP-MS for differentiation of white latex paints. 


\section{Chapter 6: Conclusions}

Although it is less sensitive than LA-ICP-MS, LIBS has been demonstrated as an effective tool for the discrimination of white latex paints via elemental analysis. Current methods, such as visual identification or FTIR, are of limited use for forensic analysis. Visual inspection is tedious and rarely results in sample matches, and other techniques like FTIR are only useful in determining the type of paint used. Elemental analysis, in contrast, provides more detailed information from elemental composition that can be used to differentiate white latex paint samples.

Previous analysis of white latex paints by LA-ICP-MS demonstrated a discriminating power of 99\% for 23 samples using Tukey's pairwise discrimination. However, mass spectrometry is expensive and requires highly skilled operators. In addition, data analysis for LA-ICP-MS is more complex and tedious than for LIBS. In contrast, Tukey's pairwise comparison of the same samples resulted in $98.2 \%$ discrimination when the results of potassium (95.6\% discrimination) and aluminum (93.5\% discrimination) as elemental ratios were combined, and LIBS correctly associated the one pair composed of the same sample. The results demonstrate only slightly lower discriminating capability, and the many benefits of LIBS make it an even more attractive technique. In contrast to LA-ICP-MS, LIBS has the advantages of lower price, ease of use, limited or no sample preparation, simpler data analysis, and faster analysis time.

In addition to the Tukey analysis, the results of PCA discrimination must also be considered. The LIBS method accounted for $98.1 \%$ of the differentiation between samples while using only five elements (with potassium as the elemental ratio), which is comparable to the $99 \%$ differentiation of LA-ICP-MS, where 32 elements needed to be 
monitored. While discriminating power and accounting for variation should not be confused with each other, the high percent account of variation using LIBS suggests that the discrimination power will also be high. This was confirmed with the HSD test using LIBS. The discriminating powers of the two techniques were very similar, and show that LIBS is an effective tool for the analysis of white latex paints.

Future work could encompass the analysis of tinted paints, which contain different pigments. For example, titanium would be an unlikely candidate as an elemental ratio in colored paints, since the high levels of titanium in white latex paints are a result of the white pigment titanium dioxide. However, potassium and/or aluminum may remain in high concentrations and provide possible elemental ratios. While spectrophotometric techniques may be sufficient for some colored samples, elemental analysis would provide additional evidence for matching unknown and known samples.

Another route for additional work would be analysis of actual field samples. Latex paints are not prepared in a scientific manner when used in home settings. This could result in heterogeneity due to paints that were not thoroughly mixed, and may require a different sampling method than the one exhibited in the presented research. Other variables could be mixing of different paints, which could occur if the user has multiple cans of similar color. Application of the paint onto walls provides for additional complexity, including thickness, paint layers, and potential contribution of drywall to the sample. Preparations of the same brand at different locations should also be examined in order to determine if there is any elemental contribution associated with variations in maintenance of the dispensing machinery. While discrimination of white latex paints has been effective in the laboratory, testing field samples will present additional challenges. 
Forensic laboratories are constantly looking for more effective methods of sample analysis, including time, ease of use, and cost of instrumentation. White latex paint has presented a challenge in the past due to the limited efficacy of visual inspection and other instrumental techniques, as well as the prohibitive cost of a mass spectrometer. The present study has shown that LIBS is a valuable alternative to these other techniques; the discriminating power is comparable to that of LA-ICP-MS, and it benefits from lower cost, speed of analysis, and simplicity of operation and data analysis. In addition, LIBS can be used for a range of other applications, such as differentiation of glass [1], paper and inks [4], and cotton. [32] In the future, forensic laboratories should consider adding LIBS instrumentation to their arsenal of analytical techniques. 


\section{References}

1. Schenk, E.R. and J.R. Almirall, Elemental analysis of glass by laser ablation inductively coupled plasma optical emission spectrometry (LA-ICP-OES). Forensic science international, 2012. 217(1-3): p. 222-228.

2. Hobbs, A.L. and J.R. Almirall, Trace elemental analysis of automotive paints by laser ablation-inductively coupled plasma-mass spectrometry (LA-ICP-MS). Analytical and Bioanalytical Chemistry, 2003. 376(8): p. 1265-1271.

3. Saferstein, R., Forensic science handbook. 1982, Englewood Cliffs, N.J: PrenticeHall.

4. Trejos, T., A. Flores, and J.R. Almirall, Micro-spectrochemical analysis of document paper and gel inks by laser ablation inductively coupled plasma mass spectrometry and laser induced breakdown spectroscopy. Spectrochimica Acta, Part B: Atomic Spectroscopy, 2010. 65B(11): p. 884-895.

5. Naes, B.E., Elemental analysis of glass and ink by laser ablation inductively coupled plasma mass spectrometry (LA-ICP-MS) and laser induced breakdown spectroscopy (LIBS). 2009.

6. Gagnon, J.V., Forensic Analysis of Latex Paint by Laser Ablation- Inductively Coupled Plasma-Mass Spectrometry, in Department of Chemistry and Biochemistry. 2006, Florida International University: Miami. p. 100.

7. Howden, C.R., R.J. Dudley, and K.W. Smalldon, The nondestructive analysis of single layered household paints using energy dispersive $x$-ray fluorescence spectrometry. J., Forensic Sci. Soc., 1977. 17(Copyright (C) 2014 American Chemical Society (ACS). All Rights Reserved.): p. 161-7.

8. Yin, L.I. and S.M. Seltzer, Pattern-recognition analysis of low-resolution X-ray fluorescence spectra. Nuclear Instruments and Methods in Physics Research Section A: Accelerators, Spectrometers, Detectors and Associated Equipment, 1990. 299(1-3): p. 571-577.

9. Si, Y., L. Hai, and J. Yang, Determination of soluble lead in paint coating on wood furniture by inductively coupled plasma atomic emission spectrometry. Huaxue Yanjiu, 2011. 22(Copyright (C) 2014 American Chemical Society (ACS). All Rights Reserved.): p. 79-81.

10. Studabaker, W.B., et al., Field turbidity method for the determination of lead in acid extracts of dried paint. J. Environ. Monit., 2010. 12(Copyright (C) 2014 American Chemical Society (ACS). All Rights Reserved.): p. 1393-1403. 
11. Asfaw, A., G. Wibetoe, and D. Beauchemin, Solid sampling electrothermal vaporization inductively coupled plasma optical emission spectrometry for discrimination of automotive paint samples in forensic analysis. J. Anal. At. Spectrom., 2012. 27(Copyright (C) 2014 American Chemical Society (ACS). All Rights Reserved.): p. 1928-1934.

12. Silva, A.R., et al., Identification and determination of resins, fillers, and pigments in white latex paints. Ecletica Quim., 2000. 25(Copyright (C) 2014 American Chemical Society (ACS). All Rights Reserved.): p. 109-122.

13. McCreight, K.W., et al., Development of low VOC additives to extend the wet edge and open time of aqueous coatings. FATIPEC Congr., 2010. 30th(Copyright (C) 2014 American Chemical Society (ACS). All Rights Reserved.): p. 293-304.

14. Madrigal, L., Next generation architectural paint applications. PPCJ, Polym. Paint Colour J., 2010. 200(Copyright (C) 2014 American Chemical Society (ACS). All Rights Reserved.): p. 20-22.

15. de Lourenco, I., Jr., et al., Fiber Bragg grating sensor to monitor stress kinetics in drying process of commercial latex paints. Sensors, 2010. 10(Copyright (C) 2014 American Chemical Society (ACS). All Rights Reserved.): p. 4761-4776.

16. Zhou, W.-h., et al., Study on the making techniques and materials of the oilpainting samples from a column in Bogda Khan Palace Museum. Neimenggu Daxue Xuebao, Ziran Kexueban, 2010. 41(Copyright (C) 2014 American Chemical Society (ACS). All Rights Reserved.): p. 522-526.

17. Ponterio, R., et al., Laser induced breakdown spectroscopy for the analysis of archaeological dyes from Licata (Sicily). Radiat. Eff. Defects Solids, 2008. 163(Copyright (C) 2014 American Chemical Society (ACS). All Rights Reserved.): p. 535-543.

18. Garcia Gimenez, R., et al., Analytical and multivariate study of roman age architectural terra cotta from northeast of Spain. Talanta, 2005. 65(Copyright (C) 2014 American Chemical Society (ACS). All Rights Reserved.): p. 861-868.

19. Miziolek, A.W., V. Palleschi, and I. Schechter, Laser Induced Breakdown Spectroscopy. 2006, Cambridge: Cambridge University Press.

20. Singh, J.P., et al., Laser-induced breakdown spectroscopy (LIBS). Anal. Bioanal. Chem., 2011. 400(Copyright (C) 2013 American Chemical Society (ACS). All Rights Reserved.): p. 3191-3192. 
21. Cremers, D.A. and L.J. Radziemski, Handbook of laser-induced breakdown spectroscopy. 2nd ed. ed. 2013, Chichester, West Sussex, U.K: John Wiley \& Sons, Ltd.

22. Aeschliman, D.B., et al., Spatially-resolved analysis of solids by laser ablationinductively coupled plasma-mass spectrometry: trace elemental quantification without matrix-matched solid standards. Journal of Analytical Atomic Spectrometry, 2003. 18(8): p. 872-877.

23. Lazic, V., et al., Quantitative elemental analyses of archaeological materials by laser induced breakdown spectroscopy (LIBS) - an overview. Proc. SPIE-Int. Soc. Opt. Eng., 2005. 5857(Copyright (C) 2014 American Chemical Society (ACS). All Rights Reserved.): p. 58570H/1-58570H/12.

24. Tognoni, E., et al., Calibration-Free Laser-Induced Breakdown Spectroscopy: State of the art. Spectrochim. Acta, Part B, 2010. 65B(Copyright (C) 2014 American Chemical Society (ACS). All Rights Reserved.): p. 1-14.

25. Cong, Z.-b., et al., Quantitative analysis of alloy steel based on laser induced breakdown spectroscopy with partial least squares method. Guangpuxue Yu Guangpu Fenxi, 2014. 34(Copyright (C) 2014 American Chemical Society (ACS). All Rights Reserved.): p. 542-547.

26. Guo, L.-b., et al., Quantitative analysis of vanadium and titanium in steel samples using LIBS. Guangpuxue Yu Guangpu Fenxi, 2014. 34(Copyright (C) 2014 American Chemical Society (ACS). All Rights Reserved.): p. 217-220.

27. Hobbs, A., Elemental Analysis of Automotive Paints by Laser AblationInductively Coupled Plasma-Mass Spectrometry (LA-ICP-MS) and Paint and Glass by Laser Induced Breakdown Spectroscopy (LIBS), in Department of Chemistry \& Biochemistry. 2003, Florida International University: Miami.

28. Li, H., et al., A kind of method of detecting full content of iron in iron ore based on laser-induced breakdown spectroscopy technology [Machine Translation]. 2014, Northwest University, Peop. Rep. China . p. 7pp.

29. Choi, D., et al., Laser-induced breakdown spectroscopy (LIBS) analysis of calcium ions dissolved in water using filter paper substrates: an ideal internal standard for precision improvement. Appl. Spectrosc., 2014. 68(Copyright (C) 2014 American Chemical Society (ACS). All Rights Reserved.): p. 198-212.

30. Lasheras, R.J., C. Bello-Galvez, and J.M. Anzano, Quantitative analysis of oxide materials by laser-induced breakdown spectroscopy with argon as an internal standard. Spectrochim. Acta, Part B, 2013. 82(Copyright (C) 2014 American Chemical Society (ACS). All Rights Reserved.): p. 65-70. 
31. Naes, B.E., et al., A comparison of laser ablation inductively coupled plasma mass spectrometry, micro X-ray fluorescence spectroscopy, and laser induced breakdown spectroscopy for the discrimination of automotive glass.

Spectrochimica Acta, Part B: Atomic Spectroscopy, 2008. 63B(10): p. 1145-1150.

32. Schenk, E.R. and J.R. Almirall, Elemental analysis of cotton by laser-induced breakdown spectroscopy. Applied Optics, 2010. 49(13): p. C153-C160.

33. Windom, B.C. and D.W. Hahn, Laser ablation-laser induced breakdown spectroscopy (LA-LIBS): A means for overcoming matrix effects leading to improved analyte response. J. Anal. At. Spectrom., 2009. 24(Copyright (C) 2014 American Chemical Society (ACS). All Rights Reserved.): p. 1665-1675.

34. Hahn, D.W., Laser-induced breakdown spectroscopy for analysis of aerosol particles: the path toward quantitative analysis. Spectroscopy (Duluth, MN, United States), 2010(Journal Article): p. 23-28.

35. Evans, E.H., et al., Advances in atomic spectrometry and related techniques. J. Anal. At. Spectrom., 2011. 26(Copyright (C) 2014 American Chemical Society (ACS). All Rights Reserved.): p. 1115-1141.

36. JMP®, Version 11. 1989-2007, SAS Institute Inc.: Cary, NC.

37. Miller, J.N., J.C. Miller, and J.C.S.f.a.c. Miller, Statistics and chemometrics for analytical chemistry. 4th ed. ed. 2000, Harlow, England; New York: Prentice Hall.

38. Abdi, H.W., L., Tukey's Honestly Significant Difference (HSD) Test. Encyclopedia of Research and Design, ed. N. Salkind. 2010, Thousand Oaks, CA: Sage.

39. Winer, B.J., Statistical principles in experimental design. 2d ed. ed. McGraw-Hill series in psychology. 1971, New York: McGraw-Hill. 